\title{
Distributed Data-based Fault Identification and Accommodation in Networked Process Systems
}

\author{
Di Penga,b, Nael H. El-Farra ${ }^{\mathrm{b}, *}$, Zhiqiang Geng ${ }^{\mathrm{a}}$, Qunxiong Zhu ${ }^{\mathrm{a}}$ \\ ${ }^{a}$ College of Information Science \& Technology \\ Beijing University of Chemical Technology, Beijing 100029, China \\ ${ }^{b}$ Department of Chemical Engineering 8 Materials Science \\ University of California, Davis, CA 95616, USA
}

\begin{abstract}
This paper presents a data-based framework for distributed actuator fault identification and accommodation in networked process systems controlled over a resource-constrained communication medium. Initially, a quasi-decentralized networked control structure is designed to stabilize the plant in the absence of faults. The structure consists of a set of local model-based control systems that communicate with one other at discrete times. An explicit characterization of the networked closed-loop stability region is obtained in terms of the update period, the accuracy of the models, and the choice of controller design parameters. To address the actuator fault identification problem, a set of local fault diagnosis agents are designed and embedded within the various subsystems. Each agent uses a moving-horizon parameter estimation scheme to estimate on-line the size and location of the local faults using the locally-sampled states and the model state estimates for the interconnected units. Potential discrepancies or ambiguities in the local fault diagnosis results, which may be caused by the strong dynamic coupling between the individual subsystems and the presence of plant-model mismatch, are reconciled by means of a fault estimation confidence interval which is obtained by analyzing the networked closed-loop dynamics at update times. Once the locations and magnitudes of the actuator faults are identified, the resulting estimates are transmitted to a higher-level supervisor to select and implement a suitable fault accommodation strategy. A number of stability-preserving fault accommodation strategies are devised, including updating the post-fault models, adjusting the controllers' parameters, or a combination of both. The selection of the appropriate fault accommodation strategy is made on the basis of the estimated fault magnitude and the characterization of the networked closed-loop stability region. Finally, the developed methodology is illustrated using a reactor-separator process example subject to both sudden and incipient control actuator faults.
\end{abstract}

Keywords: Networked control, fault identification, fault accommodation, quasi-decentralized control, large-scale systems, process networks.

\section{Introduction}

Chemical plants are large-scale dynamical systems that typically involve a large number of distributed interconnected subsystems that are tightly integrated through material, energy and information flows and recycle. Control and supervision of such large-scale systems are fundamental problems that encompass a host of theoretical and practical challenges. These problems have received significant attention in process control over the past few decades and have motivated many research studies on the analysis and design of distributed and supervisory control systems for process networks (see, for example, [5, 6, 16, 17, 19, 23, 27] for some results and references in this area). In addition to these advances, research efforts within process

*Author of correspondence. Tel: (530)754-6919, Fax: (530) 752-1031, Email: nhelfarra@ucdavis.edu

Preprint submitted to Chem. Eng. Sci.

February 16, 2015

(C) 2015. This manuscript version is made available under the Elsevier user license http://www.elsevier.com/open-access/userlicense/1.0/ 
control have recently begun to address the new challenges emerging from the integration of networked control systems in process operations. These efforts have been motivated by a number of recent developments in the field, including the shift in industrial operation towards sensor and distributed control systems that are accessed over shared communication networks rather than dedicated links $[10,11,28]$; as well as the drive towards expanding the traditional process control an operations paradigm in the direction of smart plant operations [4].

A key feature of smart plant operations is the use of advanced cyber-infrastructure and communication technologies (such as wireless sensor networks) to achieve tight real-time integration of control, computing and communication at different levels of the plant, in order to realize the desired environmental, health and safety targets. In this context, efforts to address problems such as network resource utilization and real-time scheduling constraints were initiated in a number of studies which led to the design of resourceaware networked plant-wide control [24], state estimation [25] and communication scheduling methods [26] that enforce the desired stability and performance properties with minimal communication requirements. A central aim of these methods is to balance the tradeoff that exists between the achievable control quality on the one hand, and the extent of network utilization by the units sharing the communication medium, on the other. To keep the necessary information transfer to a minimum, a model-based quasi-decentralized control structure is used, whereby a set of dynamic models are included within each local control system to provide estimates of the states of the interconnected units. These estimates, which are used to generate the local control action when communication is suspended, are then corrected or updated using the actual state measurements whenever communication between the different subsystems is restored.

The quasi-decentralized control structure offers a compromise between the complexity and lack of flexibility associated with centralized control structures on the one hand, and the performance limitations of purely decentralized schemes, on the other. However, the problems of fault diagnosis and handling were not explicitly addressed in the networked control system design. These are important problems that require special attention in the context of large-scale process networks. Specifically, due to the interconnections between the plant units through mass and energy flows and recycle, and the resulting dynamic coupling between the various component subsystems, the adverse effects of local faults in a given subsystem may propagate to the rest of the plant and potentially cause failure at the plant level, if not explicitly accounted for. Timely identification and handling of faults are therefore essential capabilities that the networked plant-wide control system needs to have.

Fault diagnosis and fault-tolerant control are fundamental problems at the interface of process control and operations, which are motivated by the need to develop autonomous and intelligent process control and monitoring systems that operate reliably in the presence of faults. Faults in dynamic processes are characterized by critical and unpredictable changes in the process dynamics resulting possibly from malfunctions in the control actuators, the measurement sensors, and/or the process equipment. Within the context of smart plant operations, fault detection and accommodation are essential tools for realizing the goal of zero-incident plant operations. While the literature on these problems is quite extensive (see, for example, $[2,8,14,15,22,29,31]$, for some results and references in this area), systematic methods for the design of fault-tolerant control systems for integrated process networks are limited at present. Notable exceptions within process control include control approaches based on distributed model predictive control formulations [3] and safe-parking frameworks [9]. These approaches, however, do not explicitly account for communication constraints in the control system design. Outside of process control research, fault detection of distributed multi-agent systems has also received some attention, including, for example, efforts to develop algorithms for distributed fault diagnosis in discrete event systems [1], design of sensor networks for fault-tolerant estimation [21], strategies for fault-tolerance in distributed systems [20], decentralized fault detection [20] and decomposition-based distributed fault detection and isolation using adaptive approximations [7]. The focus of these studies, however, has been primarily on the fault diagnosis task. Also, networked communication issues between the component subsystems were not explicitly considered in the problem formulation.

Motivated by these considerations, we present in this work an approach that combines process data and process models to achieve distributed identification and accommodation of control actuator faults in networked process systems controlled over a resource-constrained communication medium. A model-based 
quasi-decentralized networked control structure containing a set of local model-based control systems that communicate with each other at discrete times is initially designed. By analyzing the behavior of the networked closed-loop system, an explicit characterization of the closed-loop stability region is obtained in terms of the network update period, the model uncertainty, the magnitudes of the faults and the controller design parameters. The fault identification mechanism involves the use of a set of local fault diagnosis agents, which are embedded in the various subsystems. A moving-horizon optimization problem is solved by each agent at each sampling time to determine on-line the size and location of the local faults using the past sampled state data and the states of the locally embedded models describing the dynamics of the interconnected units. An estimation confidence interval of the actual fault parameter is obtained to resolve the ambiguities introduced by the strong dynamic coupling between the individual subsystems and the presence of plant-model mismatch. Once the faults are identified, the estimated magnitudes of the faults are sent to a higher-level supervisor, which uses this information, together with the characterization of the networked closed-loop stability region, to determine the appropriate fault accommodation strategy. The fault accommodation strategies include updating the post-fault models in the plant subsystems and/or switching to a new set of stabilizing controller parameters.

The rest of the paper is organized as follows. In section 2, mathematical preliminaries are presented describing the class of networked process systems considered, as well as an overview of the problem formulation. Section 3 then focuses on the design of the model-based quasi-decentralized networked controller and presents an analysis of the networked closed-loop stability properties. The fault identification and fault accommodation strategies are then presented in Section 4. Finally, the proposed framework is illustrated in Section 5 using a networked reactor-separator process example, and conclusions are given in 6.

\section{Preliminaries}

\subsection{Class of process networks}

In this study, we focus on large-scale networked process systems comprised of $n$ interconnected subsystems or units, where each subsystem is modeled by a continuous-time linear system, and described by the following state space representation:

$$
\begin{aligned}
\dot{x}_{1} & =A_{1} x_{1}+B_{1} \theta_{1} u_{1}+\sum_{j=2}^{n} A_{1 j} x_{j} \\
\dot{x}_{2} & =A_{2} x_{2}+B_{2} \theta_{2} u_{2}+\sum_{j=1, j \neq 2}^{n} A_{2 j} x_{j} \\
& \vdots \\
\dot{x}_{n} & =A_{n} x_{n}+B_{n} \theta_{n} u_{n}+\sum_{j=1}^{n-1} A_{n j} x_{j}
\end{aligned}
$$

where $x_{i}:=\left[x_{i}^{(1)} x_{i}^{(2)} \cdots x_{i}^{\left(p_{i}\right)}\right]^{T} \in \mathbb{R}^{p_{i}}$ denotes the vector of process state variables associated with the $i$-th unit, $p_{i}$ is the number of state variables in the $i$-th unit. $u_{i}:=\left[u_{i}^{(1)} u_{i}^{(2)} \cdots u_{i}^{\left(q_{i}\right)}\right]^{T} \in \mathbb{R}^{q_{i}}$ denotes the vector of manipulated inputs associated with the $i$-th unit, $x^{T}$ denotes the transpose of $x$, and $A_{i}, B_{i}$ and $A_{i j}$ are constant matrices of appropriate dimensions. The interconnection term $A_{i j} x_{j}$, where $i \neq j$, describes how the $j$-th unit influences the dynamics of the $i$-th unit in the plant. $\theta_{i}:=\operatorname{diag}\left\{\theta_{i}^{(1)} \theta_{i}^{(2)} \ldots \theta_{i}^{\left(q_{i}\right)}\right\}$ is a diagonal fault matrix that accounts for the presence of control actuator faults. Each element of the fault matrix $\theta_{i}$ takes values between zero and one, where zero denotes total actuator failure while one denotes the fault-free state of the actuator. This representation essentially models the effect of an actuator fault in terms of the level of degradation in the controller authority caused by the fault.

\subsection{Overview of problem formulation and solution approach}

We consider a quasi-decentralized networked control architecture similar to the one considered in [24]. In this architecture, each unit in the plant has a local control system with its sensors and actuators connected 
to the local controller via dedicated links, and the units exchange measurements of the local states over a shared resource-constrained communication network that constrains the rate of information transfer. The sensor-controller and controller-actuator communications within each local unit are considered to occur more frequently without delays due to the presence of the dedicated link, while the information transfer between the different subsystems over the plant-wide network is assumed to take place only at discrete times. During periods of communication suspension, a set of prediction models are used within each subsystem to provide estimates of the missing states, and the states of each model are updated using the actual state measurements transmitted when communication is restored.

Given this networked control structure, the problems under consideration include (1) how to identify the size and location of control actuator faults within each subsystem in a timely fashion, despite the dynamic coupling between the various subsystems and the inherent uncertainties in the prediction models, and (2) how to devise a suitable fault accommodation strategy that preserves the stability of the overall networked closed-loop system in the presence of faults. To address these problems, we consider a distributed monitoring architecture in which a local fault diagnosis agent is embedded within each subsystem. Each agent is designed to provide an on-line estimation of the size and location of the local fault parameters by solving a moving horizon parameter estimation problem at each sampling time using the past locally-sampled states and the states of the prediction models. To overcome the possible ambiguities in the fault diagnosis results caused by the dynamic coupling and plant-model mismatch, an analysis of the model estimation errors at communication times is conducted to estimate, for each fault parameter, a confidence interval where the possible actual values of the fault are expected to lie given the available bounds on the model uncertainty. Once the locations and magnitudes of the faults are identified, a higher-level supervisor receives the resulting estimates and implements the desired fault accommodation strategy to maintain stability of the individual units and the overall plant. The choice of the fault accommodation strategy is made based on the position of the operating point with respect to the networked closed-loop stability region. A schematic representation of this networked fault identification, accommodation and control architecture is shown in Fig.1. To illustrate the main ideas and simplify the presentation of the results, we will focus in this work on the full state feedback problem where all the states of all the units are available as measurements. Extensions to the output feedback case are possible and the subject of other research work.

\section{Quasi-decentralized networked control system design}

The objectives of this section are (1) to design a quasi-decentralized networked controller that enforces closed-loop stability, and (2) to explicitly characterize the networked closed-loop stability region in terms of the fault magnitudes as well as the various control and communication system design parameters.

\subsection{Distributed feedback controller synthesis}

To realize the desired networked quasi-decentralized control structure, we begin by synthesizing, for each subsystem, a state feedback controller that stabilizes the local states at the origin in the absence of communication limitations (i.e., when the sensors of each unit transmit their data continuously to the control systems of the other units). We consider control laws of the general form:

$$
u_{i}(x)=K_{i} x_{i}+\sum_{j=1, j \neq i}^{n} K_{i j} x_{j}
$$

where $K_{i}$ is the local feedback gain responsible for stabilizing the $i$-th subsystem in the absence of interconnections, and $K_{i j}$ is the controller gain that compensates for the effect of the $j$-th subsystem on the dynamics of the $i$-th unit.

The implementation of this controller as written requires continuous availability of the state measurements. Unlike the local measurements which are available continuously through a dedicated link, the measurements from the other units are only available at discrete time instances. To reduce the information transfer between the units over the communication network, dynamic models of the interconnected subsystems are embedded within the local control system of each unit to provide estimates of the states of its 
neighbouring units when measurements are not broadcast over the network. Based on these models, the local control law for each unit is computed and implemented as follows:

$$
u_{i}(t)=K_{i} x_{i}(t)+\sum_{j=1, j \neq i}^{n} K_{i j} \widehat{x}_{j}^{i}(t), i \in\{1,2, \cdots, n\}
$$

where $\widehat{x}_{j}^{i}$ is an estimate of $x_{j}$ provided by a model of unit $j$ embedded in the local control system of unit $i$, and is given by:

$$
\begin{aligned}
& \dot{\hat{x}}_{j}^{i}(t)=\widehat{A}_{j} \widehat{x}_{j}^{i}(t)+\widehat{B}_{j} \widehat{\theta}_{j}^{i} \widehat{u}_{j}^{i}(t)+\widehat{A}_{j i} x_{i}(t)+\sum_{l=1, l \neq i, l \neq j}^{n} \widehat{A}_{j l} \widehat{x}_{l}^{i}(t), \quad t \in\left(t_{k h}, t_{(k+1) h}\right) \\
& \widehat{u}_{j}^{i}(t)=K_{j} \widehat{x}_{j}^{i}(t)+K_{j i} x_{i}(t)+\sum_{l=1, l \neq i, l \neq j}^{n} K_{j l} \widehat{x}_{l}^{i}(t), \quad t \in\left(t_{k h}, t_{(k+1) h}\right) \\
& \widehat{x}_{j}^{i}\left(t_{k h}\right)=x_{j}\left(t_{k h}\right), \quad j \in\{1, \cdots, n\}, \quad j \neq i, k \in\{0,1,2, \cdots\}
\end{aligned}
$$

Referring to Eq.4, $\widehat{A}_{j}, \widehat{B}_{j}, \widehat{A}_{j l}$ are constant matrices that approximate the actual plant matrices, i.e.,

$$
\widehat{A}_{j}=A_{j}+\Delta A_{j}, \widehat{B}_{j}=B_{j}+\Delta B_{j}, \widehat{A}_{j l}=A_{j l}+\Delta A_{j l}
$$

where $\Delta A_{j}, \Delta B_{j}$, and $\Delta A_{j l}$ represent the plant-model mismatch which is assumed to be bounded, and $\widehat{\theta}_{j}^{i}:=\operatorname{diag}\left\{\widehat{\theta}_{j}^{i(1)} \widehat{\theta}_{j}^{i(2)} \ldots \widehat{\theta}_{j}^{i\left(q_{j}\right)}\right\}$ is a diagonal matrix containing estimates of the fault parameters in unit $j, \theta_{j}$. The model states $\widehat{x}_{j}^{i}, j \neq i$ are used to calculate the control action for unit $i$ during periods of network communication suspension. The state of the model $\widehat{x}_{j}^{i}$ is then updated using the actual state $x_{j}$ when it becomes available from the network at time $t=t_{k h}$, where $t_{k h}$ is a transmission time, $h$ is the total number of sampling intervals between two consecutive transmission times, and $\zeta=t_{(k+1) h}-t_{k h}$ is the transmission/update period which is assumed to be constant and the same for all the units, i.e., all state measurements are available to each controller every $\zeta$ units of time.

\subsection{Networked closed-loop stability analysis}

To analyze closed-loop stability, we begin by defining the model estimation error as:

$$
e_{j}^{i}:=x_{j}-\widehat{x}_{j}^{i}, \quad j \neq i
$$

where $e_{j}^{i}$ represents the difference between the state of the $j$-th unit and the state of its model used in the local control system of the $i$-th unit. Note that because measurements of $x_{i}$ are assumed to be continuously available to the local control system of the $i$-th unit, we always have $e_{i}^{i}=0$. Introducing the augmented error and state vectors:

$$
\begin{aligned}
& \mathbf{e}_{j}:=\left[\left(e_{j}^{1}\right)^{T}\left(e_{j}^{2}\right)^{T} \cdots\left(e_{j}^{n}\right)^{T}\right]^{T} \\
& \mathbf{e}:=\left[\begin{array}{llll}
\mathbf{e}_{1}^{T} & \mathbf{e}_{2}^{T} & \cdots & \mathbf{e}_{n}^{T}
\end{array}\right]^{T} \\
& \mathbf{x}:=\left[\begin{array}{llll}
x_{1}^{T} & x_{2}^{T} & \cdots & x_{n}^{T}
\end{array}\right]^{T} \\
& \xi(t):=\left[\begin{array}{lll}
\mathbf{x}^{T}(t) & \mathbf{e}^{T}(t)
\end{array}\right]^{T},
\end{aligned}
$$

it can be shown that the overall closed-loop system of Eqs.1-4 can be formulated as a combined discretecontinuous system of the general form:

$$
\begin{aligned}
\dot{\xi}(t) & =\Lambda \xi(t), \quad t \in\left[t_{k h}, t_{(k+1) h}\right) \\
\Lambda & =\left[\begin{array}{ll}
\Lambda_{11} & \Lambda_{12} \\
\Lambda_{21} & \Lambda_{22}
\end{array}\right] \\
\xi\left(t_{k h}\right) & =\left[\begin{array}{ll}
\mathbf{x}^{T}\left(t_{k h}\right) & \mathbf{0}
\end{array}\right]^{T}, \quad k \in\{0,1,2, \cdots\}
\end{aligned}
$$


where the state $\mathbf{x}$ evolves continuously in time, and the estimation error $\mathbf{e}\left(t_{k h}\right)$ is reset to zero at the update time $t_{k h}$. Referring to Eq.7, the matrices $\Lambda_{11}, \Lambda_{12}, \Lambda_{21}$, and $\Lambda_{22}$ are linear combinations of the plant matrices, $A_{i}, B_{i}$, and $A_{i j}$; the model matrices, $\widehat{A}_{i}, \widehat{B}_{i}$, and $\widehat{A}_{i j}$; the controller matrices, $K_{i}$ and $K_{i j}$; and the fault matrices, $\theta_{i}$ and $\widehat{\theta}_{j}^{i}$. The explicit forms of these matrices can be obtained by substituting Eqs.3-4 into Eq.(1).

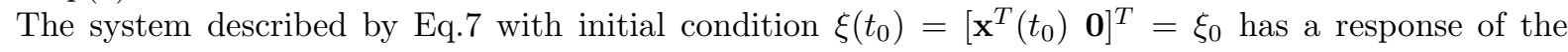
following form:

$$
\xi(t)=e^{\Lambda\left(t-t_{k h}\right)} M^{k} \xi_{0}, \text { for } t \in\left[t_{k h}, t_{(k+1) h}\right)
$$

where $M$ is given by:

$$
M=\left[\begin{array}{ll}
I_{11} & O_{12} \\
O_{21} & O_{22}
\end{array}\right] e^{\Lambda \zeta}\left[\begin{array}{ll}
I_{11} & O_{12} \\
O_{21} & O_{22}
\end{array}\right]
$$

where the null matrix $O$ resets the estimation error e to zero at each update time. Based on this solution, it can be shown (see [24]) for a proof that a necessary and sufficient condition for exponential closed-loop stability of the plant under the quasi-decentralized networked structure is to have all the eigenvalues of the test matrix $M$ strictly inside the unit circle, i.e.,

$$
\lambda_{\max }\left(M\left(\Delta A_{i}, \Delta B_{i}, \Delta A_{i j}, K_{i}, K_{i j}, \zeta, \theta_{i}, \widehat{\theta}_{i}\right)\right)<1
$$

where $\lambda_{\max }(M)$ is the maximum eigenvalue magnitude of $M$. It can be seen from Eq.10 that the maximum eigenvalue magnitude is dependent on the size of the plant-model mismatch, the choice of controller gains, the update period, and the magnitude of the actuator faults. All these factors are tied together through the stability condition of Eq.10 which can be used to examine and quantify the various interdependencies between these factors and how they influence closed-loop stability. For instance, if the update period, the controllers' gains, and the model uncertainty are fixed, one can use Eq.10 to determine the range of actuator fault magnitudes that can be tolerated without causing instability. This characterization is also important since it forms the basis upon which a suitable fault accommodation strategy can be chosen and implemented. This will be discussed in more detail in Section 4.2.

\section{Distributed fault identification and accommodation}

Having designed and characterized the stability properties of the quasi-decentralized networked control structure, we turn in this section to the design of an integrated fault identification and accommodation strategy that uses a data-based approach, together with process models, to detect, isolate, estimate and compensate for faults taking place in the control actuators within the plant subsystems. To this end, we initially synthesize, for each subsystem, a local fault diagnosis agent that estimates on-line the size of the local fault parameter based on the local states and the estimated states of the neighboring subsystems obtained from the available models. Once the faults are identified and their magnitudes are estimated, a stability-based fault accommodation algorithm, which prescribes the appropriate fault correction measures that need to be taken following fault identification, is developed.

\subsection{Data-based fault identification strategy}

In order to estimate the actuator fault parameters within a given subsystem, a local model-based fault diagnoser of the following form is used to provide estimates of the local states:

$$
\begin{aligned}
& \dot{\tilde{x}}_{i}^{i}(t)=\widehat{A}_{i} \widetilde{x}_{i}^{i}(t)+\widehat{B}_{i} \theta_{i}^{*} \widetilde{u}_{i}(t)+\sum_{j=1, j \neq i}^{n} \widehat{A}_{i j} \widehat{x}_{j}^{i}(t), \quad i \in\{1,2, \cdots, n\} \\
& \widetilde{u}_{i}(t)=K_{i} \widetilde{x}_{i}^{i}(t)+\sum_{j=1, j \neq i}^{n} K_{i j} \widehat{x}_{j}^{i}(t)
\end{aligned}
$$


where $\tilde{x}_{i}^{i}$ is the state of the $i$-th diagnoser agent which provides an estimate of $x_{i}, \widehat{x}_{j}^{i}$ is a copy of the state of the $j$-th model embedded in the $i$-th subsystem, $\widetilde{u}_{i}$ is the estimated local control action computed using a combination of the local diagnoser state and the estimated model states of the interconnected subsystems, $\theta_{i}^{*}$ is a diagonal matrix which provides an estimate of $\theta_{i}$, where each diagonal element in this matrix, $\theta_{i}^{*(q)}$, $q \in\left\{1,2, \cdots, q_{i}\right\}$, represents the estimated value of the fault in the $q$-th actuator in the $i$-th subsystem to be determined through the fault identification process. Note that, similar to the model-based controller considered in Eqs.3-4, due to the dynamic coupling between the plant subsystems and the desire to limit the frequency of information exchange over the network, each local fault diagnoser must rely on models of the interconnected subsystems that provide estimates of the interactions between the various subsystems at times when information exchange between the units is suspended. The states of these models need to be updated using the actual state measurements whenever information is exchanged between the plant subsystems.

Since the local state measurements are typically available only at discrete times due to measurement sampling, the continuous-time form of the diagnoser in Eq.11 needs to be transformed into a discrete-time form so that it is more suitable for sampled-data fault identification. To this end, the following augmented vector is defined to lump together the state of the local diagnoser and the states of all the auxiliary models embedded in the $i$-th subsystem:

$$
\widetilde{\mathbf{x}}^{i}:=\left[\left(\widehat{x}_{1}^{i}\right)^{T}\left(\widehat{x}_{2}^{i}\right)^{T}\left(\widetilde{x}_{i}^{i}\right)^{T} \cdots\left(\widehat{x}_{n}^{i}\right)^{T}\right]^{T}
$$

Combining Eqs.3-4 with Eq.11, and using the definition of the augmented diagnoser state in Eq.12, the dynamics of the $i$-th fault diagnoser can be represented in the following compact form:

$$
\dot{\tilde{\mathbf{x}}}^{i}(t)=F^{i} \widetilde{\mathbf{x}}^{i}(t), i \in\{1,2, \cdots, n\}
$$

where the matrix $F^{i}$ is a linear combination of $\widehat{A}_{i}, \widehat{B}_{i}, \widehat{A}_{i j}, \widehat{\theta}_{i}$ and $\theta_{i}^{*}$, and the state $\widetilde{\mathbf{x}}^{i}$ is subject to the following update laws:

$$
\begin{aligned}
\widetilde{x}_{i}^{i}\left(t_{l}\right) & =x_{i}\left(t_{l}\right), l \in\{0,1,2, \cdots\} \\
\widehat{x}_{j}^{i}\left(t_{k h}\right) & =x_{j}\left(t_{k h}\right), j \in\{1,2, \cdots, n\}, j \neq i, k \in\{0,1,2, \cdots\}
\end{aligned}
$$

where $t_{l}$ is a sampling time and $t_{k h}$ is a transmission time. The first update law in Eq.14 ensures that the state of the local diagnoser is constantly updated and corrected using the available local state at each sampling time, while the second update law ensures that the states of the models providing the local diagnoser with estimates of the interactions between the plant subsystems are updated using the actual state measurements whenever communication is allowed to take place over the plant-wide network. The second update law is the same as the one introduced earlier in Eq.4 for updating the control models.

Based on the continuous-time form of Eq.13 and the update laws of Eq.14, the following discrete-time representation of the dynamics of the local fault diagnoser (and the auxiliary models embedded in the $i$-th subsystem) can be obtained:

$$
\widetilde{\mathbf{x}}^{i}[l+1]=G^{i}\left(\theta_{i}^{*}\right) \widetilde{\mathbf{x}}^{i}[l], i \in\{1,2, \cdots, n\}
$$

where $\widetilde{\mathbf{x}}^{i}[l]=\widetilde{\mathbf{x}}^{i}\left(t_{l}\right)$ is the discrete augmented diagnoser state for unit $i, G^{i}\left(\theta_{i}^{*}\right)=e^{F^{i} \omega}$, and $\omega=t_{l+1}-t_{l}$ is the sampling period which is considered to be much smaller than the update period $\zeta$. This is consistent with the assumption that the local state is available more frequently (or continuously in the limit as $\omega$ tends to zero) than the states of the interconnected subsystems which must be transmitted through the plant-wide network.

Based on Eq.15, the discrete state of the $i$-th fault diagnoser, $\widetilde{x}_{i}^{i}$, can be calculated from:

$$
\widetilde{x}_{i}^{i}[l+1]=G_{i i}^{i}\left(\theta_{i}^{*}\right) x_{i}[l]+\sum_{j=1, j \neq i}^{n} G_{i j}^{i}\left(\theta_{i}^{*}\right) \widehat{x}_{j}^{i}[l]
$$

where $G_{i j}^{i}\left(\theta_{i}^{*}\right)$ denotes the element in $i$-th row and $j$-th column of $G^{i}\left(\theta_{i}^{*}\right)$, and $\widetilde{x}_{i}^{i}[l]=x_{i}[l]$ per the update law of Eq.14. 
By comparing the state estimates generated by the local fault diagnoser with the actual local states over a certain horizon length, the local fault parameter in each unit can be estimated by solving online a moving-horizon least-square optimization problem, where the cost function to be minimized is the square of the model prediction error, and is defined as follows:

$$
\begin{aligned}
J\left(\Upsilon_{l}, \theta_{i}^{*}\right) & =\sum_{p=l}^{l-N_{I}+1}\left\|x_{i}[p+1]-\widetilde{x}_{i}^{i}[p+1]\right\|^{2} \\
& =\sum_{p=l}^{l-N_{I}+1}\left\|x_{i}[p+1]-G_{i i}^{i}\left(\theta_{i}^{*}\right) x_{i}[p]-\sum_{j=1, j \neq i}^{n} G_{i j}^{i}\left(\theta_{i}^{*}\right) \widehat{x}_{j}^{i}[p]\right\|^{2}
\end{aligned}
$$

where $x_{i}[p]=x_{i}\left(t_{p}\right)$ is the sampled local state at time $t_{p}, \Upsilon_{l}$ denotes the set of current and past local state measurements and estimated model states of the interconnected units:

$$
\Upsilon_{l}=\left\{\left(x_{i}[l-p], \widehat{x}_{j}^{i}[l-p]\right) \mid p \in\left\{0,1, \cdots, N_{I}-1\right\}\right\}
$$

and $N_{I}$ is the number of sampled state measurements used for fault identification. $N_{I}$ also represents the horizon over which the online parameter estimation problem is solved. Based on Eqs.17-18, the value of $\theta_{i}^{*}$ at any sampling time $t_{l}$ can be determined by solving the following optimization problem:

$$
\min _{\theta_{i}^{*}} J\left(\Upsilon_{l}, \theta_{i}^{*}\right)
$$

Based on the solution to this optimization problem, a fault in the $q$-th actuator in the $i$-th subsystem can be detected whenever a noticeable change in the resulting estimate of $\theta_{i}^{*(q)}$ is observed. It should be noted, however, that the optimal solution $\theta_{i}^{*}$ for each unit will not, in general, be exactly the same as the actual fault parameter $\theta_{i}$, due to (1) the inherent mismatch between the plant and prediction model dynamics (i.e., the fact that $A_{i} \neq \widehat{A}_{i}, A_{i j} \neq \widehat{A}_{i j}$, and $B_{i} \neq \widehat{B}_{i}$ ), and (2) the discrepancies between the measured states and estimated model states of the interconnected units resulting from the use of different fault parameter estimates during periods of communication suspension. These discrepancies occur, for example, when a fault in the $j$-th subsystem occurs and influences the evolution of the state $x_{j}$, but the prediction model state $\widehat{x}_{j}^{i}$ used in the $i$-th subsystem has not yet received this fault information during communication suspension. Since the value of $\theta_{i}$ is an indicator of both the faults' locations and magnitudes, the estimation of the real fault parameter $\theta_{i}$ is crucial for the system's ability to take the appropriate fault accommodation measure. To resolve the possible discrepancies between $\theta_{i}^{*}$ and $\theta_{i}$ introduced by the plant-model mismatch, we seek in the following development to obtain, for each fault, a fault estimation confidence interval which provides upper and lower bounds for the fault magnitude given available bounds on the model uncertainty. This confidence interval is then used in Section 4.2 to implement the fault accommodation logic. To illustrate how this confidence interval can be estimated, we begin by analyzing the dynamics of the local state prediction error, $\widetilde{e}_{i}^{i}:=x_{i}-\widetilde{x}_{i}^{i}$, which represents the error between the state of the $i$-subsystem of Eq.1 and the state of the diagnoser model of Eq.11. The error dynamics are given by:

$$
\begin{aligned}
\dot{\tilde{e}}_{i}^{i}(t) & =\dot{x}_{i}(t)-\dot{\widetilde{x}}_{i}^{i}(t) \\
& =\left[\Delta A_{i}+\left(B_{i} \theta_{i}-\widehat{B}_{i} \theta_{i}^{*}\right) K_{i}\right] x_{i}(t)+\left[\widehat{A}_{i}+\widehat{B}_{i} \theta_{i}^{*} K_{i}\right] \widetilde{e}_{i}^{i}(t) \\
& +\sum_{j=1, j \neq i}^{n}\left[\Delta A_{i j}+\left(B_{i} \theta_{i}-\widehat{B}_{i} \theta_{i}^{*}\right) K_{i j}\right] x_{j}(t) \\
& +\sum_{j=1, j \neq i}^{n}\left[\widehat{A}_{i j}-\left(B_{i} \theta_{i}-\widehat{B}_{i} \theta_{i}^{*}\right) K_{i j}\right] e_{j}^{i}(t), i \in\{1,2, \cdots, n\}
\end{aligned}
$$

Taking the update laws of Eq.14 into consideration, we have that at any transmission time, $t_{k h}$, the following conditions hold: (1) the state of each model is updated using the actual state measurements, i.e., $\widehat{x}_{j}^{i}\left(t_{k h}\right)=$ 
$x_{j}\left(t_{k h}\right)$ and, therefore, $e_{j}^{i}\left(t_{k h}\right)=0$ for $j \in\{1, \cdots, n\}$, and (2) the state of the local $i$-th diagnoser is updated using the locally sampled state of the $i$-th subsystem, i.e., $\widetilde{x}_{i}^{i}\left(t_{k h}\right)=x_{i}\left(t_{k h}\right)$ and, therefore, $\widetilde{e}_{i}^{i}\left(t_{k h}\right)=0$. These conditions imply that the error dynamics at the transmission time are governed by:

$$
\begin{aligned}
\dot{\tilde{e}}_{i}^{i}\left(t_{k h}\right) & =\Delta A_{i} x_{i}\left(t_{k h}\right)+\sum_{j=1, j \neq i}^{n} \Delta A_{i j} \widehat{x}_{j}^{i}\left(t_{k h}\right) \\
& +\widehat{B}_{i}\left(\theta_{i}-\theta_{i}^{*}\right)\left[K_{i} x_{i}\left(t_{k h}\right)+\sum_{j=1, j \neq i}^{n} K_{i j} \widehat{x}_{j}^{i}\left(t_{k h}\right)\right] \\
& -\Delta B_{i} \theta_{i}\left[K_{i} x_{i}\left(t_{k h}\right)+\sum_{j=1, j \neq i}^{n} K_{i j} \widehat{x}_{j}^{i}\left(t_{k h}\right)\right]
\end{aligned}
$$

where we have used the fact that $\widehat{B}_{i}=B_{i}+\Delta B_{i}$. By inspecting the right-hand side of Eq.21, it can be verified that, with the exception of $\theta_{i}$, all the other terms are either known or have known bounds. Terms that are known include the local state and model states at the transmission time, $x_{i}\left(t_{k h}\right)$ and $\widehat{x}_{j}^{i}\left(t_{k h}\right)$, the model matrices, $\widehat{B}_{i}$, the controller gain matrices, $K_{i}$ and $K_{i j}$, and the estimated fault matrix, $\theta_{i}^{*}$, obtained as the solution of the moving-horizon optimization problem which is carried out at each sampling time. Terms that have known bounds include the matrices describing the model uncertainty, $\Delta A_{i}, \Delta B_{i}$ and $\Delta A_{i j}$. Therefore, if the time-derivative of the local state prediction error, $\dot{\tilde{e}}_{i}^{i}\left(t_{k h}\right)$, can be estimated, an estimate of the range of possible values for $\theta_{i}$ can be obtained. To this end, using a finite-difference approximation scheme, an estimate of the time-derivative of the local state prediction error at the transmission time can be obtained as follows:

$$
\widetilde{e}_{i}^{i}\left(t_{k h+1}^{-}\right)=\widetilde{e}_{i}^{i}\left(t_{k h}\right)+\omega \dot{\tilde{e}}_{i}^{i}\left(t_{k h}\right)+o\left(\omega^{2}\right)
$$

where $\widetilde{e}_{i}^{i}\left(t_{k h}\right)=0$ due to Eq.14, $\widetilde{e}_{i}^{i}\left(t_{k h+1}^{-}\right)=x_{i}\left(t_{k h+1}^{-}\right)-\widetilde{x}_{i}^{i}\left(t_{k h+1}^{-}\right)=x_{i}\left(t_{k h+1}\right)-\widetilde{x}_{i}^{i}\left(t_{k h+1}^{-}\right)$due to the continuity of $x_{i}$ in time, $t_{k h+1}$ is the first sampling time following the $k h$-th transmission time, and the notation $t_{k h+1}^{-}$is used to indicate the time right before the local state diagnoser is updated using the available sampled local state. Note from the definition of the error $e_{i}^{i}\left(t_{k h+1}^{-}\right)$that it can be computed since both $x_{i}\left(t_{k h+1}\right)$ and $\widetilde{x}_{i}^{i}\left(t_{k h+1}^{-}\right)$are known. Therefore, combining Eq.21 and Eq.22, the actual fault parameter $\theta_{i}$ can be locally estimated at time $t_{k h+1}$ by solving the following optimization problem:

$$
\min _{\theta_{i}}\left\|\widetilde{e}_{i}^{i}\left(t_{k h+1}^{-}\right)-\omega \dot{\widetilde{e}}_{i}^{i}\left(t_{k h}\right)\right\|^{2}
$$

where $\dot{\tilde{e}}_{i}^{i}\left(t_{k h}\right)$ is given by Eq.21. Note that due to the dependence of the error dynamics on the uncertain parameters (i.e., $\Delta A_{i}, \Delta A_{i j}$ and $\Delta B_{i}$ ), the above optimization problem will in general yield a different solution for different values of the parametric uncertainty. While some physical process parameters may be unknown, it is reasonable to assume that a bounding interval, which captures the range of possible values for a given parameter, is known. In this case, the collection of solutions that result from solving Eq.23 over this interval represent a fault estimation confidence interval, which we denote by $\Psi\left(\theta_{i}^{*(q)}\right):=\left[\grave{\theta}_{i, \min }^{(q)}, \grave{\theta}_{i, \max }^{(q)}\right]$. This interval represents all possible values that the fault parameter for a given actuator, $\theta_{i}^{(q)}$, can take for a given $\theta_{i}^{*(q)}$ and given bounds on the size of the plant-model mismatch. In other words, for a given solution to the optimization problem of Eqs.17-19 and given bounds on the size of the plant-model mismatch, one can conclude with confidence that the actual fault magnitude lies within $\Psi\left(\theta_{i}^{*(q)}\right)$. The fault estimation confidence interval plays an important role in the fault identification scheme since it provides an estimate of the size of the fault and can also be used for fault detection. Specifically, under fault-free conditions, one can obtain a confidence interval for each actuator in a given subsystem, $\Psi_{h}\left(\theta_{i}^{*(q)}\right)$, which captures the range of possible values of $\theta_{i}^{(q)}$ that satisfy Eq.23. The lower bound of $\Psi_{h}\left(\theta_{i}^{*(q)}\right)$, which we denote by $\grave{\theta}_{i}^{(q), \text { th }}$, can be used as a threshold for monitoring the health status of the $q$-th actuator in unit $i$. In particular, a 
fault in the $i$-th unit can be declared when the upper bound of the resulting confidence interval, $\Psi\left(\theta_{i}^{*(q)}\right)$, is less than $\grave{\theta}_{i}^{(q) \text {,th }}$. The absence of any overlap between the actual and fault-free confidence intervals indicates that the observed variations in the fault parameter estimate cannot simply be explained by the plant-model mismatch alone, and hence must be due to an actual fault.

Remark 1: It should be noted that the sampling period $\omega$ is allowed to be much smaller than the update period $\zeta$, in order to make the approximation error $o\left(\omega^{2}\right)$ in Eq.22 close to zero, and ensure that Eq.23 provides a reasonably accurate approximation of the real fault parameter. This consideration is also consistent with the assumption that the transmission of the local state information between the sensors and controller within a given subsystem occurs more frequently than information transmission between the different plant subsystems.

Remark 2: Unlike the moving-horizon optimization problem of Eq.19, which is solved at each sampling time to track possible changes in $\theta_{i}^{*}$ over time, the optimization problem of Eq. 23 is solved only at the update (transmission) time. Due to the use of a communication network to transfer information between the plant subsystems, the fault estimation confidence interval $\Psi\left(\theta_{i}^{*(q)}\right)$ can be estimated only when the measured states are transmitted through network. Therefore, although an actuator fault may occur between transmission times (i.e., $\left.T_{f} \in\left(t_{(k-1) h}, t_{k h}\right)\right)$, the earliest time that the fault magnitude can be estimated with some certainty is one sampling time after the next transmission time, i.e., $T_{d}=t_{k h+1}$. The time instances at which the moving horizon optimization, measurement transmission, and fault estimation occur are depicted in the time-line shown in Fig.2.

Remark 3: The developed data-based fault identification method can be used not only to detect faults but also to identify which actuator has malfunctioned. This is important when multiple actuators are used for control but there is not an appropriate fault isolation mechanism to determine where the fault is.

Remark 4: In the special case when no plant-model mismatch is present, the solution to the optimization problem of Eqs.17-19 provides an exact estimate of the actual fault, i.e., $\theta_{i}^{*}=\theta_{i}$. This can be justified by examining Eq.21 and Eq.23 when $\Delta A_{i}=0, \Delta B_{i}=0$ and $\Delta A_{i j}=0$. In this case, Eq.19, which is used for estimating the confidence interval, reduces to:

$$
\min _{\theta_{i}}\left\|\widetilde{e}_{i}^{i}\left(t_{k h+1}^{-}\right)-\omega B_{i}\left(\theta_{i}-\theta_{i}^{*}\right)\left[K_{i} x_{i}\left(t_{k h}\right)+\sum_{j=1, j \neq i}^{n} K_{i j} \widehat{x}_{j}^{i}\left(t_{k h}\right)\right]\right\|^{2}
$$

Furthermore, due to the absence of plant-model mismatch, we have $x_{i}(t)=\widetilde{x}_{i}^{i}(t)$ which implies that the local state prediction error is zero, i.e., $\widetilde{e}_{i}^{i}\left(t_{k h+1}^{-}\right)=0$. Under these conditions it can be verified that the minimum of the cost function in Eq.24 is attained when $\theta_{i}^{*}=\theta_{i}$. This implies that the fault estimation confidence interval, $\Psi\left(\theta_{i}^{*(q)}\right)$, collapses to a single point $\grave{\theta}_{i}^{(q)}$ which is the same as the value of the actual fault parameter $\theta_{i}^{(q)}$. In this case, a fault taking place in the $q$-th actuator of the $i$-th unit can be declared when $\grave{\theta}_{i}^{(q)}<1$ (where $q \in\left\{1,2, \cdots, q_{i}\right\}$ ), and, in addition, the magnitude of the fault can be determined as $\grave{\theta}_{i}^{(q)}$.

\subsection{Fault accommodation strategies}

Once the location and magnitude of the fault in a given subsystem are identified, the fault parameter estimate $\theta_{i}^{*(q)}$ and the associated estimation confidence interval $\Psi\left(\theta_{i}^{*(q)}\right)$ are transmitted to a higher-level supervisor. On the basis of these estimates, the supervisor determines an appropriate corrective action to accommodate the fault and maintain closed-loop stability. Possible fault accommodation measures include (1) updating the post-fault control models in the other plant subsystems (i.e., using $\theta_{i}^{*}$ to update the fault parameter estimate of the $i$-th model in all the interconnected units $\widehat{\theta}_{i}^{j}$, for $\left.j \in\{1,2, \cdots, n\}, j \neq i\right),(2)$ switching to a new set of stabilizing controller gains for the $i$-th subsystem, and (3) a combination of model updates and controller gain adjustment. In some cases, when a partial fault is not significant enough to destroy the stability properties of the plant, switching to a new control configuration is often not necessary. Considering this situation, we consider below a stability-based fault accommodation logic which is formulated in Algorithm 1. The key idea is to maintain the pre-fault control configuration (including the dynamic 
control models, the controller gains and the choice of manipulated variables) as long as all the elements of the confidence interval $\Psi\left(\theta_{i}^{*}\right)$, for $i \in\{1,2, \cdots, n\}$, satisfy the stability condition of Eq.10; otherwise, the local control system should switch to a new controller configuration to guarantee the stability of the closed-loop system.

\section{Algorithm 1.}

1. choose an update period $\zeta$ that satisfies the stability condition of Eq.10, set $\widehat{\theta}_{i}^{j}=I_{q_{i} \times q_{i}}$, for $i, j \in$ $\{1,2, \cdots, n\}, j \neq i$, and set $l=0$

2. solve the optimization problem of Eqs.17-19 to estimate $\theta_{i}^{*}$ for $i \in\{1,2, \cdots, n\}$

3. if $t_{l}=t_{k h+1}$ and $k \in\{0,1,2, \cdots\}$, estimate $\Psi\left(\theta_{i}^{*(q)}\right)$ for each $\theta_{i}^{*(q)}$ using Eq.23 and Eq.21

4. if for any $\vartheta_{i}^{(q)} \in \Psi\left(\theta_{i}^{*(q)}\right)$, the condition of Eq.10 is violated with $\theta_{i}^{(q)}=\vartheta_{i}^{(q)}$

5. update $\widehat{\theta}_{i}^{j(q)}$ using $\theta_{i}^{*(q)}$, for $j \in\{1,2, \cdots, n\}, j \neq i$

6. if for all $\varsigma_{i}^{(q)} \in \Psi\left(\theta_{i}^{*(q)}\right)$, the stability condition of Eq.10 is satisfied with $\theta_{i}^{(q)}=\varsigma_{i}^{(q)}$ and $\widehat{\theta}_{i}^{j(q)}=\theta_{i}^{*(q)}$

7. GOTO step 14 directly

8. else if for some $K_{i}^{*}$ and $K_{i j}^{*}$ the stability condition of Eq.10 is satisfied

9. update $K_{i}, K_{i j}$ using $K_{i}^{*}, K_{i j}^{*}$ and GOTO step 14

10. else

11. replace the $q$-th actuator in the $i$-th unit with a new actuator that satisfies the stability condition of Eq.10, set $\widehat{\theta}_{i}^{j(q)}=1$ and GOTO step 14

12. end if

13. else

14. set $l=l+1$ and GOTO step 2

15. end if

Remark 5: Given that the implementation of the fault accommodation strategies requires knowledge of the fault estimation confidence interval, and the fact that this interval can be estimated only at transmission times (see Remark 2), there will in general be some lag between the time that a fault occurs and is detected and the time that corrective action is taken. This time lag can be minimized by choosing a sufficiently small update period which would ensure a more timely fault estimation and accommodation. However, reducing the update period invariably increases network resources utilization. The selection of the update period should, therefore, be made in a way that balances the inherent tradeoff between the desired fault-tolerant control performance, on the one hand, and the extent of network resource utilization, on the other. Another approach to ensure timely fault recovery is to employ an event-triggered scheme in which the update rate can be adjusted in response to fault events. For some recent results on networked control using an eventtriggered communication strategy and its potential advantages in handling unanticipated disturbance, the reader is referred to $[12,30]$.

Remark 6: Even though Algorithm 1 considers adjusting only the local controller gains, $K_{i}$ and $K_{i j}$, to accommodate a given local fault in the $i$-th unit, it is possible for the supervisor to consider adjusting the controller gains of the other subsystems as well before switching to other locally available actuators. The logic in this case would be the same as the one considered in Algorithm 1 which is to choose controller gains that satisfy the stability condition of Eq.10. Note that following the adjustment of the controller gains in any given unit, the corresponding model of this unit embedded in the other subsystems must be updated accordingly to capture the post fault accommodation conditions and ensure closed-loop stability.

Remark 7: The fault accommodation algorithm described in this section is not limited to the case of a single fault in a given unit, but can be applied to the case of multiple faults taking place either concurrently or at different times in different subsystems. In these cases, the solutions of the optimization problems of Eqs.17-19 and Eqs.21-22 determine the locations and possible magnitudes of the faulty actuators. These faults can then be accommodated following a logic similar the one described in Algorithm 1, such as updating all $\widehat{\theta}_{i}^{j(q)}$ associated with the faulty actuators, $\theta_{i}^{*(q)}$, or using new feedback gains, or a combination of both. 
Furthermore, the proposed fault identification and accommodation approach can be applied to the case of faults with time-varying magnitudes. In this case, by solving the moving-horizon estimation problem at each sampling time, the fault diagnosis agent can track the evolution of the fault and implement the appropriate fault accommodation strategy which would bring the operating point back inside the stability region at any time that the fault pushes it outside. This case is demonstrated in Section 5.3.

Remark 8: As mentioned in Remark 4, when a perfect model is used for control and fault identification, the fault accommodation strategy described in Algorithm 1 is simplified since the stability condition of Eq.10 needs to be verified for a single point, $\grave{\theta}_{i}^{(q)}$, rather than for all possible points in the estimation confidence interval $\Psi\left(\theta_{i}^{*(q)}\right)$.

Remark 9: The proposed networked fault-tolerant control structure brings together two key components: quasi-decentralized control and data-based fault identification and accommodation. The integration of these two components impacts the way in which each component is implemented within the overall structure. For example, in conventional quasi-decentralized control [24], where no fault-tolerance capabilities are explicitly included, only the model states are updated at transmission times. In the integrated networked and fault-tolerant control structure, however, model parameters may also require updating at transmission times to ensure fault accommodation. Also, the use of quasi-decentralized networked control impacts the implementation of the fault accommodation approach by limiting the availability of state measurements from the plant to a given subsystem (which are needed to estimate the fault magnitude), thus resulting in delayed fault accommodation (see Remark 2).

\section{Simulation study: Application to a reactor-separator plant}

To illustrate the design and implementation of the proposed fault identification and accommodation strategy, we consider a process with two well-mixed continuous stirred tank reactors (CSTRs) followed by a flash tank separator as shown in Fig.3. In the two CSTRs, two irreversible elementary exothermic reactions

of the forms $A \stackrel{k_{1}}{\longrightarrow} B$ and $A \stackrel{k_{2}}{\longrightarrow} C$ take place in parallel, where the reactant $A$ yields a desired product $B$ and an undesired by-product $C$, while the solvent $D$ does not react. This process operates as follows: The reactant $A$ is fed to CSTR 1 at flow rate $F_{10}$, concentration $C_{A 10}$ and temperature $T_{10}$ and the two products $B$ and $C$ are formed; the effluent of CSTR 1 at flow rate $F_{1}$ enters CSTR 2 together with another inlet stream containing pure $A$ at flow rate $F_{20}$, concentration $C_{A 20}$ and temperature $T_{20}$ and the reactant is further reacted; the effluent of CSTR 2 at flow rate $F_{2}$ then enters the flash tank where the overhead vapor is condensed and enters CSTR 1 as a recycle stream at flow rate $F_{r}$. A jacket is used to remove or provide heat to each unit of this process. Under standard modeling assumptions, the plant dynamics which can be 
derived from energy and material balances take the following form:

$$
\begin{aligned}
& \dot{T}_{1}=\frac{F_{10}}{V_{1}}\left(T_{10}-T_{1}\right)+\frac{F_{r}}{V_{1}}\left(T_{3}-T_{1}\right)-\frac{\Delta H_{1}}{\rho c_{p}} k_{1} e^{\frac{-E_{1}}{R T_{1}}} C_{A 1}-\frac{\Delta H_{2}}{\rho c_{p}} k_{2} e^{\frac{-E_{2}}{R T_{1}}} C_{A 1}+\frac{Q_{1}}{\rho c_{p} V_{1}} \\
& \dot{C}_{A 1}=\frac{F_{10}}{V_{1}}\left(C_{A 10}-C_{A 1}\right)+\frac{F_{r}}{V_{1}}\left(C_{A r}-C_{A 1}\right)-k_{1} e^{\frac{-E_{1}}{R T_{1}}} C_{A 1}-k_{2} e^{\frac{-E_{2}}{R T_{1}}} C_{A 1} \\
& \dot{C}_{B 1}=\frac{-F_{10}}{V_{1}} C_{B 1}+\frac{F_{r}}{V_{1}}\left(C_{B r}-C_{B 1}\right)+k_{1} e^{\frac{-E_{1}}{R T_{1}}} C_{A 1} \\
& \dot{C}_{C 1}=\frac{-F_{10}}{V_{1}} C_{C 1}+\frac{F_{r}}{V_{1}}\left(C_{C r}-C_{C 1}\right)+k_{2} e^{\frac{-E_{2}}{R T_{1}}} C_{A 1} \\
& \dot{T}_{2}=\frac{F_{1}}{V_{2}}\left(T_{1}-T_{2}\right)+\frac{F_{20}}{V_{2}}\left(T_{20}-T_{2}\right)-\frac{\Delta H_{1}}{\rho c_{p}} k_{1} e^{\frac{-E_{1}}{R T_{2}}} C_{A 2}-\frac{\Delta H_{2}}{\rho c_{p}} k_{2} e^{\frac{-E_{2}}{R T_{2}}} C_{A 2}+\frac{Q_{2}}{\rho C_{p} V_{2}} \\
& \dot{C}_{A 2}=\frac{F_{1}}{V_{2}}\left(C_{A 1}-C_{A 2}\right)+\frac{F_{20}}{V_{2}}\left(C_{A 20}-C_{A 2}\right)-k_{1} e^{\frac{-E_{1}}{R T_{2}}} C_{A 2}-k_{2} e^{\frac{-E_{2}}{R T_{2}}} C_{A 2} \\
& \dot{C}_{B 2}=\frac{F_{1}}{V_{2}}\left(C_{B 1}-C_{B 2}\right)-\frac{F_{20}}{V_{2}} C_{B 2}+k_{1} e^{\frac{-E_{1}}{R T_{2}}} C_{A 2} \\
& \dot{C}_{C 2}=\frac{F_{1}}{V_{2}}\left(C_{C 1}-C_{C 2}\right)-\frac{F_{20}}{V_{2}} C_{C 2}+k_{2} e^{\frac{-E_{2}}{R T_{2}}} C_{A 2} \\
& \dot{T}_{3}=\frac{F_{2}}{V_{3}}\left(T_{2}-T_{3}\right)-\frac{H_{\mathrm{vap}} F_{r}}{\rho c_{p} V_{3}}+\frac{Q_{3}}{\rho c_{p} V_{3}} \\
& \dot{C}_{A 3}=\frac{F_{2}}{V_{3}}\left(C_{A 2}-C_{A 3}\right)-\frac{F_{r}}{V_{3}}\left(C_{A r}-C_{A 3}\right) \\
& \dot{C}_{B 3}=\frac{F_{2}}{V_{3}}\left(C_{B 2}-C_{B 3}\right)-\frac{F_{r}}{V_{3}}\left(C_{B r}-C_{B 3}\right) \\
& \dot{C}_{C 3}=\frac{F_{2}}{V_{3}}\left(C_{C 2}-C_{C 3}\right)-\frac{F_{r}}{V_{3}}\left(C_{C r}-C_{C 3}\right)
\end{aligned}
$$

where all process variables are defined in Table 1. In the separator, the relative volatility of each species is assumed to remain constant within the operating temperature range of the flash tank, and the amount of reacting material in the separator is assumed to be negligible. Thus, the concentrations of $A, B$ and $C$ in the recycle are given by:

$$
\begin{gathered}
C_{A r}=\frac{\alpha_{A} C_{A 3}}{K}, C_{B r}=\frac{\alpha_{B} C_{B 3}}{K}, C_{C r}=\frac{\alpha_{C} C_{C 3}}{K} \\
K=\alpha_{D}+\left(\alpha_{A}-\alpha_{D}\right) C_{A 3} \frac{M W_{A}}{\rho}+\left(\alpha_{B}-\alpha_{D}\right) C_{B 3} \frac{M W_{B}}{\rho}+\left(\alpha_{C}-\alpha_{D}\right) C_{C 3} \frac{M W_{C}}{\rho}
\end{gathered}
$$

For the process parameter values given in Table 2, it can be verified that the plant has an unstable steadystate which is given in Table 2. The control objective is the stabilization of the plant states at this steadystate by manipulating the rates of heat transfer for the three units. To this end, linearizing the plant model around the unstable steady state yields a system of the form of Eq.1 which is used to design the quasidecentralized networked controller of Eqs.3-4, leading to closed-loop dynamics of the form of Eq.7), where the dimensionless state vector $x_{i}$ and manipulated input $u_{i}$ for the $i$-th unit, $i \in\{1,2,3\}$, are defined by:

$$
\begin{aligned}
& x_{i}=\left[\frac{T_{i}-T_{i}^{s}}{T_{i}^{s}} \frac{C_{A i}-C_{A i} s}{C_{A i}^{s}} \frac{C_{B i}-C_{B i}^{s}}{C_{B i}^{s}} \frac{C_{C i}-C_{C i}^{s}}{C_{C i}^{s}}\right]^{\mathrm{T}}, i \in\{1,2,3\} \\
& u_{1}=Q_{1}, \quad u_{2}=Q_{2}, \quad u_{3}=Q_{3}
\end{aligned}
$$

The explicit forms of the various matrices are omitted for brevity. Based on this model, a model-based controller of the form of Eq.3 is applied to achieve the stabilization objective in absence of faults, subject to a choice of the update period $\zeta=0.1 \mathrm{hr}$ which satisfies the stability condition of Eq.10. Furthermore, a measurement sampling period of $\omega=0.005 \mathrm{hr}$ is chosen and a horizon of $N_{I}=10$ is used for fault identification. 


\subsection{Handling actuator faults under no plant-model mismatch}

To illustrate some of the key ideas in the proposed fault identification and accommodation scheme, we first consider the case when a fault takes place in the control actuator of the first CSTR, and a perfect model is used for control and fault identification purposes (the case of multiple actuator faults is considered in Section 5.4). We begin by analyzing the dependence of closed-loop stability on the magnitude of the actuator fault, the choice of the fault parameter estimate used in the control models, and the choice of the local controller gains.

Fig.4(a) is a contour plot depicting the dependence of the maximum eigenvalue magnitude of the stability test matrix $M, \lambda_{\max }(M)$, on $\theta_{1}$ and $\widehat{\theta}_{1}^{2}$, where $\theta_{1}$ is the size of the actual fault, and $\widehat{\theta}_{1}^{2}$ is the estimated value of the fault used in the control models embedded in the second and third units (i.e., we consider $\widehat{\theta}_{1}^{2} \equiv \widehat{\theta}_{1}^{3}$ ). The uncolored area which lies inside the unit contour line represents the stability region. It can be seen that as $\theta_{1}$ decreases from 1 (i.e., the fault severity increases), the stability region shrinks and the range of feasible values for $\widehat{\theta}_{1}^{2}$ becomes smaller. Recall that $\widehat{\theta}_{i}^{j}$ are possible fault accommodation parameters which may or may not need to adjusted to maintain closed-loop stability. The significance of Fig.4(a) in the context of fault accommodation is that it can be used to determine if, and how, the control model parameters can be updated to accommodate the fault.

Fig.4(b) is another contour plot which shows the dependence of $\lambda_{\max }(M)$ on $\theta_{1}$ and $\alpha_{1}$, where $\alpha_{1}$ is a tuning parameter that is introduced to allow varying the local controller gains, i.e., we set $K_{i}^{*}=\alpha_{i} K_{i}$. The uncolored area inside the unit contour line represents the stability region in this case. It can be seen that as $\alpha_{1}$ increases (i.e., the strength of the controller gain increases), the stability region increases and the range of tolerable faults that do not compromise closed-loop stability increases.

To confirm the predictions of Fig.4 and illustrate how the fault identification and fault accommodation strategies are implemented, we consider a number of simulation scenarios involving faults with different magnitudes. In each scenario, the fault is assumed to take place at $t=0.33 \mathrm{hr}$. The first case considers a relatively minor, but sudden fault with $\theta_{1}=0.9$ at $t=0.33 \mathrm{hr}$. The fault profile is shown by the solid line in Fig.5(a). The dashed line in the figure represents the evolution of $\theta_{1}^{*}$ obtained by solving the optimization problem of Eqs.17-19 at each sampling time. It can be seen that at $t=0.405 \mathrm{hr}$ (which represents the first sampling instance after the first transmission time since the fault occurs) the magnitude of $\theta_{1}^{*}$ drops from 1 to 0.9105 and settles close to 0.9 after a few sampling times. Due to the absence of any plant-model mismatch in this case, the fault estimation confidence interval of $\Psi\left(\theta_{1}^{*}\right)$ contains only a single estimate, $\grave{\theta}_{1}$, which is the same as the actual fault. Note that the short transient before $\theta_{1}^{*}$ settles at the actual fault value is due to the fact that the data set used for on-line optimization contains a mixture of sampled states from both the pre-fault and post-fault operating regimes. Based on the fault identification result, the supervisor needs to determine whether fault accommodation is necessary. As can be observed from Fig.5(b), the fault causes a shift in the operating point from the initial point with $\left(\theta_{1}=1, \widehat{\theta}_{1}^{2}=1\right)$ to point A with $\left(\theta_{1}=\grave{\theta}_{1}\right.$, $\left.\widehat{\theta}_{1}^{2}=1\right)$ which is still located inside the stability region. This suggests that closed-loop stability is not compromised under this fault and that fault accommodation by means of an update of the control model parameters, $\widehat{\theta}_{1}^{2}$ and $\widehat{\theta}_{1}^{3}$, is not necessary. This result is further confirmed by the closed-loop temperature and reactant concentration profiles for the first CSTR shown in Figs.5(c)-(d).

The second case considers a sudden, but more significant, fault where $\theta_{1}$ changes from 1 to 0.7 at $t=0.33$ hr. The profiles of the actual fault and its estimated value from the online optimization are shown by the solid and dashed profiles in Fig. 6(a), respectively. It can be seen that, after a short transient, $\theta_{1}^{*}$ settles at 0.7172 at $t=0.405 \mathrm{hr}$, and that $\grave{\theta}_{1}=0.7$.

Fig.6(b) shows that the fault shifts the operating point from the initial point at $\left(\theta_{1}=1, \widehat{\theta}_{1}^{2}=1\right)$ to point $\mathrm{B}$ at $\left(\theta_{1}=\grave{\theta}_{1}, \widehat{\theta}_{1}^{2}=1\right)$ which lies outside the stability region. This implies that the fault will be destabilizing if not properly compensated for. To avoid instability, the post-fault operating point needs to be pushed back inside the stability region. As shown in Fig.6(b), this can be achieved by updating the fault parameter estimates $\widehat{\theta}_{1}^{2}$ and $\widehat{\theta}_{1}^{3}$ using the optimized estimate, $\theta_{1}^{*}$, which moves the operating point to point $\mathrm{B}^{*}$ inside the stable region. This prediction is further confirmed by the closed-loop temperature and reactant concentration profiles for the first CSTR in Figs.6(c)-(d), which show that, if the post-fault control model parameters are updated, closed-loop stability is maintained. 
When the severity of the fault is sufficiently large such that updating the control model parameters cannot compensate for the destabilizing tendency due to the fault, the supervisor needs to implement an alternative strategy for fault accommodation by adjusting some of the other degrees of freedom related to the stability condition of Eq.10. To show this scenario, we consider a third case in which a sudden fault occurs such that $\theta_{1}$ drops from 1 to 0.3 at $t=0.33 \mathrm{hr}$. The malfunction of the actuator is detected at $t=0.405 \mathrm{hr}$, at which time $\theta_{1}^{*}=0.3455$ and $\grave{\theta}_{1}=0.3$. It can bee seen from Fig.7(a) that the post-fault operating point is well within the unstable region and therefore fault accommodation is necessary to preserve closed-loop stability. It can also be seen that updating the model parameters using $\theta_{1}^{*}$ is not sufficient to push the operating point back into the stability region, since point $\mathrm{C}^{*}$ is still outside the stability region. To ensure closed-loop stability, the local controller gain in the first CSTR is increased by a factor of 3.33 (i.e., $\alpha_{1}=3.33$ ), which pushes the operating point back inside the stability region as can be seen from direction of the arrows in Fig.7(b). Note that the uncolored area in Fig.7(b) is the stability region in terms of $\theta_{1}$ and $\alpha_{1}$ when $\widehat{\theta}_{1}^{2}=\widehat{\theta}_{1}^{3}=\theta_{1}^{*}$, and, therefore, point $\mathrm{C}^{*}$ in Fig.7(a) is exactly the same as point $\mathrm{C}^{*}$ in Fig.7(b). Figs.7(c)-(d) show the resulting closed-loop temperature and reactant concentration profiles for the first CSTR when the model parameters are updated and the controller gain is subsequently adjusted.

Notice that while it would have been possible to accommodate this fault by choosing a sufficiently small value for $\theta_{1}^{2}$ that would push the operating point inside the stability region, the post-fault model updating strategy becomes completely infeasible for faults with $\theta_{1}<0.2$ for which no stabilizing values of $\theta_{1}^{2}$ exist. In such cases, one must consider alternative measures such as controller gain modifications.

\subsection{Handling actuator faults under plant-model mismatch}

In this section, we consider the situation where the models used for fault identification and control are not exactly the same as the plant. For simulation purposes, we introduce a plant-model mismatch in the heats of reactions, and consider $\Delta H_{1}^{\text {nom }}=\Delta H_{1}+\delta_{1} \Delta H_{1}, \Delta H_{2}^{\text {nom }}=\Delta H_{2}+\delta_{2} \Delta H_{2}$, where $\Delta H_{1}^{\text {nom }}$ and $\Delta H_{2}^{\text {nom }}$ are the nominal values used in the models, and $\delta_{1}=\delta_{2}=0.05$.

Some model parameters in the plant might have measurement errors because they are difficult to be accurately measured, but bounds on the measurement errors can be obtained. Based on such bounds, the range of plant-model mismatch can be estimated. In this study, we consider that the parametric uncertainties in the heats of reactions have the following bounds, where $\delta_{1} \in\left[-0.1 \Delta H_{1}^{\text {nom }}, 0\right]$ and $\delta_{2} \in\left[-0.1 \Delta H_{2}^{\text {nom }}, 0\right]$. As observed from Eq.25, the parameters $\Delta H_{1}$ and $\Delta H_{2}$ only influence the states of two reactors, which means that model uncertainty appears only in the first two units, while the model of the separator is exactly the same as the process. When the closed-loop system is run under fault-free conditions, the following fault detection thresholds are obtained for the three units: $\grave{\theta}_{1}^{\text {th }}=0.9761, \grave{\theta}_{2}^{\text {th }}=0.9491$, and $\grave{\theta}_{3}^{\text {th }}=1$, where the thresholds for the first units are less than 1 due to the model uncertainty.

To apply the developed fault identification and accommodation strategy, we first consider a case where a sudden fault that changes $\theta_{1}$ from 1 to 0.9 takes place at $t=0.33 \mathrm{hr}$. Fig.8(a) shows the profiles of the actual fault (solid) and its estimated value (dashed) based on the moving horizon optimization of Eq.1719. The fault is detected at $t=0.405 \mathrm{hr}$ and the solution of the optimization problem yields an estimate of $\theta_{1}^{*}=0.9205$ at that time, which breaches the fault detection alarm threshold. Due to the presence of parametric uncertainty, a fault estimation confidence interval is also calculated after each transmission time to try to estimate the actual size of the fault. These intervals are shown by the dashed vertical lines, where the circle markers denote the upper and lower bounds. At $t=0.405$, the estimation confidence interval was found to be $\Psi\left(\theta_{1}^{*}\right)=[0.8756,0.9248]$.

Fig.8(b) shows the stability region (uncolored zone) in the $\left(\theta_{1}, \widehat{\theta}_{1}^{2}\right)$ parametric space with $\alpha_{1}=1$. When compared with the stability region in Fig.4(a) for the uncertainty-free case, it is evident that the size of the stability region shrinks due to the influence of model uncertainty. More importantly, Fig.8(b) shows that the lower bound of the fault estimation interval A falls outside the stability region, which implies that the detected fault could be destabilizing if no corrective action is taken. As a result, the control model parameters $\widehat{\theta}_{1}^{2}$ and $\widehat{\theta}_{1}^{3}$ need to be updated using $\theta_{1}^{*}$. This fault accommodation strategy pushes the entire fault estimation confidence interval fully into the stability region as shown by the interval $\mathrm{A}^{*}$ to maintain closed-loop stability. The prediction of Fig.8(b) is further confirmed by the closed-loop temperature and 
reactant concentration profiles for the first CSTR in Figs.8(c)-(d) which show that closed-loop stability is maintained when the control models are updated.

We consider next the case when a more significant fault, where $\theta_{1}$ changes from 1 to 0.5 , occurs at $t=0.33$ $\mathrm{hr}$. This fault is detected at $t=0.405 \mathrm{hr}$ at which time the solution to the optimization problem of Eqs.1719 yields $\theta_{1}^{*}=0.5387$ and the estimation confidence interval is found to be $\Psi\left(\theta_{1}^{*}\right)=[0.4653,0.5362]$. From Fig.9(a), it can be seen that the entire estimation interval falls outside the stability region, and therefore fault accommodation is necessary in this case. However, updating the control model parameters using $\theta_{1}^{*}$, i.e., setting $\widehat{\theta}_{1}^{2}=\widehat{\theta}_{1}^{3}=0.5387$, does not shift the estimation interval into the stable region, since the lower bound of the new estimation interval $\mathrm{D}^{*}$ is still outside the stable region, implying that the fault may still destroy the stability of the closed-loop system. In order to maintain closed-loop stability, an alternative strategy is needed, and we consider adjusting the local controller gain. As shown in Fig. 9(b), which depicts the stability region (uncolored zone) in the $\left(\theta_{1}, \alpha_{1}\right)$ parametric space, increasing the local controller gain by a factor of 2 moves the post fault operating point inside the stability region. The effectiveness of this accommodation strategy is confirmed by the closed-loop temperature and reactant concentration profiles for the first CSTR shown in Figs.9(c)-(d) which show that the closed-loop system returns to the desired steady-state successfully.

\subsection{Handling gradual actuator faults under plant-model mismatch}

The faults considered so far have been assumed to occur abruptly. In this section, we consider the case of gradual faults that increase in severity over time. A common way to model such faults is the following logistic function [18] which we use to represent the time evolution of the fault in the control actuator of the first CSTR:

$$
\theta_{i}=a+\frac{1-a}{1+\exp \left(\frac{(t-c)}{b}\right)}
$$

where $a, b$, and $c$ are constant parameters, which, for the simulation study, are chosen to be $a=0.3, b=0.05$ hr and $c=0.33 \mathrm{hr}$.

Fig.10 shows the on-line estimation of $\theta_{1}$ for this gradual fault case. It can be seen that the estimate $\theta_{1}^{*}$ decreases slowly at the beginning, and then decreases quickly to closely track and follow the decreasing speed of $\theta_{1}$, and lastly settles close to a final value of 0.3 . Table 3 records the corresponding fault estimation confidence intervals of $\theta_{1}$ at times $0.205 \mathrm{hr}, 0.305 \mathrm{hr}, 0.405 \mathrm{hr}, 0.505 \mathrm{hr}$ and $0.605 \mathrm{hr}$. We can see that the malfunction of the actuator begins to be detected at $t=0.305 \mathrm{hr}$ because the upper limit of the confidence interval at that time $\Psi\left(\theta_{1}^{*}\right)$ is smaller than the alarm threshold $\left(\grave{\theta}_{1}^{\text {th }}=0.9761\right)$ obtained from the fault-free analysis.

Based on the values of $\theta_{1}^{*}$ and $\Psi\left(\theta_{1}^{*}\right)$, Fig.11 shows how the fault accommodation logic is implemented. At time $0.305 \mathrm{hr}$, it can be seen from Fig.11(a) that after the fault happens and is detected, the fault estimation interval lies outside the stability region (position B), so the model parameters $\widehat{\theta}_{1}^{2}$ and $\widehat{\theta}_{1}^{3}$ need to be reset at the value of $\theta_{1}^{*}$ to push the estimation interval back into the stability region (position $\mathrm{B}^{*}$ ). As the fault continues to evolve, and its magnitude increases, the fault estimation interval moves gradually outside the stability region again (position $\mathrm{C}$ ). At time $0.405 \mathrm{hr}$, the value of $\theta_{1}^{*}=0.4839$ and the estimation interval $\Psi\left(\theta_{1}^{*}\right)=[0.4036,0.4799]$. It can be seen from Fig.11(a) that in this case, however, even after updating the model parameters $\left(\widehat{\theta}_{1}^{2}=\widehat{\theta}_{1}^{3}=0.4839\right)$, the new estimation interval still lies outside the stability region (position $\mathrm{C}^{*}$ ). To prevent instability, we switch to an alternative fault accommodation strategy in which the local controller gain is increased by a factor of 2.5 , which moves the fault estimation interval completely inside the stability region in the $\left(\theta_{1}, \alpha_{1}\right)$ parametric space (position $\mathrm{C}^{* *}$ ) as can be seen from the direction of the arrows in Fig.11(b). After reconfiguring the controller, the stability region in the $\left(\theta_{1}, \widehat{\theta}_{1}^{2}\right)$ parametric space is changed as shown in Fig.11(c) due to the change in $\alpha_{1}$. As the fault continues to evolve, we find that at time $0.505 \mathrm{hr}$, the fault estimation interval is once again outside the stability region (position D). However, after updating the model parameters, the lower bound of the new estimation interval $\left(\Psi\left(\theta_{1}^{*}\right)=[0.2946,0.3512], \widehat{\theta}_{1}^{2}=\widehat{\theta}_{1}^{3}=0.3548\right)$ is still outside the stability region (position $\mathrm{D}^{*}$ ), and as a result, the controller gain is adjusted again and increased further by a factor of 1.5 to maintain closed-loop 
stability. This adjustment pushes the fault estimation interval back into the stability region in the $\left(\theta_{1}, \alpha_{1}\right)$ parametric space as shown by the direction of the arrows in Fig.11(d). Note that the stability regions in Fig.11(b) and Fig.11(d) are obtained for different values of $\theta_{1}^{2}$, and are therefore different. Following the implementation the above sequence of fault accommodation measures, the plant is successfully stabilized at the desired steady-state, as can be seen from the closed-loop concentration and rate of heat transfer profiles for the first CSTR shown in Fig.12.

\subsection{Handling multiple actuator faults under plant-model mismatch}

Because the co-occurrence probability of multiple faults is small [13], we consider in this section a case where multiple faults take place consecutively in different units. The first fault is introduced in the control actuator of the first CSTR at $t=0.33 \mathrm{hr}$ with $\theta_{1}=0.5$, and then a second fault in the control actuator of the separator is introduced at $t=1.03 \mathrm{hr}$ with $\theta_{3}=0.6$.

As observed from the fault estimation profiles in Fig.13(a), the first fault is detected at $t=0.405 \mathrm{hr}$ at which time the value of $\theta_{1}^{*}$ is 0.5387 and the fault estimation interval is found to be $\Psi\left(\theta_{1}^{*}\right)=[0.4653,0.5362]$. Prior to the time that the second fault occurs, this case is exactly the same as the one considered in Fig.9, where a fault in the first CSTR occurs at $t=0.33 \mathrm{hr}$ with $\theta_{1}=0.5$. Therefore, the fault accommodation strategy used to handle this fault is the same, and includes first updating the post-fault model parameters with $\hat{\theta}_{1}^{2}=\widehat{\theta}_{1}^{3}=0.5387$ and then adjusting the local controller gain of the first CSTR (these steps are shown in Figs.9(a)-(b)).

When the second fault happens, it can be seen from Fig.13(b) that this fault is detected at $t=1.105 \mathrm{hr}$ when the optimization-based fault parameter estimate drops suddenly, and the solution of the optimization problem of Eqs.17-19) yields $\theta_{3}^{*}=0.5787$. Due to the absence of parametric uncertainty in the dynamic model of the separator, the estimation interval of the second fault $\Psi\left(\theta_{3}^{*}\right)$ contains only a single point which corresponds to the actual fault, $\grave{\theta}_{3}=0.6$. Based on the stability region plot in Fig.14(a), it can be seen that the post-fault operating point $\left(\theta_{3}=\grave{\theta}_{3}, \widehat{\theta}_{3}^{1}=1\right)$ is located inside the stability region; and as a result, the closed-loop stability can be maintained without taking any accommodation measures. These results are further confirmed by the closed-loop temperature and concentration profiles for unit 1 and unit 3 shown in Figs. 14(b)-(d).

\section{Conclusion}

In this work, we presented an integrated data-based and model-based methodology for fault identification and accommodation in networked process systems with multiple interconnected subsystems controlled over a resource-limited communication medium. The developed methodology is based on a quasi-decentralized networked control structure in which each subsystem in the plant has a local model-based control system that communicates with the rest of the plant at discrete times. Fault identification was carried out in each subsystem via a local fault diagnosis agent that solves a moving-horizon least-square parameter estimation problem to estimate on-line the size of the local faults at each sampling time. By analyzing the dynamics of the local state prediction error at communication times, a fault estimation confidence interval was introduced to help resolve possible ambiguities in the fault estimation resulting from the strong dynamic coupling between the subsystems and the presence of plant-model mismatch. Based on the estimated magnitudes of the faults, a number of stability-based fault accommodation strategies were discussed, including updating the post-fault control models and adjusting the controller design parameters. An algorithm for deciding which accommodation strategy is feasible and appropriate for a given fault situation was developed. Finally, the results were illustrated through an application of the developed methodology to a simulated chemical plant example, subject to both abrupt and gradual control actuator faults, and shown to enforce the desired fault-tolerant control performance.

\section{Acknowledgement}

Financial support, in part by the National Science Foundation, NSF-CBET-1438456, and by the National Natural Science Foundation of China, No.61104131 and No.6137416, is gratefully acknowledged. 


\section{References}

[1] Baroni, P., Lamperti, G., Pogliano, P., Zanella, M., 1999. Diagnosis of large active systems. Artif. Intell. 110, 135-189.

[2] Blanke, M., Kinnaert, M., Lunze, J., Staroswiecki, M., 2003. Diagnosis and fault-tolerant control. Springer, Berlin, Germany.

[3] Chilin, D., Liu, J., Davis, J. F., Christofides, P. D., 2012. Data-based monitoring and reconfiguration of a distributed model predictive control system. Int. J. Rob. \& Non. Contr. 22, 68-88.

[4] Christofides, P. D., Davis, J. F., El-Farra, N. H., 2007. Smart plant operations: Vision, progress and challenges. AICHE J. 53, 2734-2741.

[5] Christofides, P. D., Liu, J., de la Pena, D. M., 2011. Networked and Distributed Predictive Control: Methods and Nonlinear Process Network Applications. Springer-Verlag, London.

[6] Cui, H., E., J., 2002. Performance limitations in decentralized control. J. Proc. Contr. 12, 485-494.

[7] Ferrari, R., Parisini, T., Polycarpou, M., 2014. Distributed fault detection and isolation of large-scale discrete-time nonlinear systems: An adaptive approximation approach. IEEE Trans. Automat. Contr. 57, 275-290.

[8] Frank, P. M., Ding, X., 1997. Survey of robust residual generation and evaluation methods in observer-based fault detection systems. J. Proc. Contr. 7, 403-424.

[9] Gandhi, R., Mhaskar, P., 2009. A safe-parking framework for plant-wide fault-tolerant control. Chem. Eng. Sci. 64, 30603071.

[10] Gupta, R. A., 2010. Networked control system: Overview and and reserach trends. IEEE Trans. Ind. Electron. 57, 25272535.

[11] Hespanha, J. P., Naghshtabrizi, P., Xu, Y., 2007. A survey of recent results in networked control systems. Proceedings of the IEEE 95, 138-162.

[12] Hu, Y., El-Farra, N. H., 2013. Quasi-decentralized output feedback model predictive control of networked process systems with forecast-triggered communication. In: Proceedings of American Control Conference. Washington, DC, pp. $2612-2617$.

[13] Iri, M., Aoki, K., O'shima, E., Matsuyama, H., 1979. An algorithm for diagnosis of system failures in chemical process. Comput. Chem. Eng. 3, 489-493.

[14] Isermann, R., 2005. Fault-Diagnosis Systems: An Introduction from Fault Detection to Fault Tolerance. Springer, Berlin, Germany.

[15] Jiang, J., Yu, X., 2012. Fault-tolerant control systems: A comparative study between active and passive approaches. Annu. Rev. in Contr. 36, 60-72.

[16] Jillson, K. R., Ydsite, B. E., 2007. Process networks with decentralized inventory and flow control. J. Proc. Contr. 17, 399-413.

[17] Jogwar, S., Baldea, M., Daoutidis, P., 2009. Dynamics and control of process networks with large energy recycle. Ind. Eng. Chem. Res. 48, 6087-6097.

[18] Lao, L., Ellis, M., Christofides, P. D., 2013. Proactive fault-tolerant model predictive control. AICHE J. 59, $2810-2820$.

[19] Lunze, J., 1992. Feedback Control of Large Scale Systems. Prentice Hall, U.K.

[20] Patton, R., Kambhampati, C., Casavola, A., Zhang, P., Ding, S., Sauter, D., 2007. A generic strategy for fault-tolerance in control systems distributed over a network. Eur. J. Contr. 13, 280-296.

[21] Staroswiecki, M., Hoblos, G., Aitouche, A., 2004. Sensor network design for fault tolerant estimation. Int. J. Adap. Contr. Sig. Proc. $18,55-72$.

[22] Steffen, T., 2005. Control reconfiguration of dynamic systems: Linear approaches and structural tests. Springer, Berlin, Germany.

[23] Stewart, B. T., Wright, S., Rawlings, J. B., 2011. Cooperative distributed model predictive control for nonlinear systems. J. Proc. Contr. 21, 698-704.

[24] Sun, Y., El-Farra, N. H., 2008. Quasi-decentralized model-based networked control of process systems. Comp. \& Chem. Eng. 32, 2016-2029.

[25] Sun, Y., El-Farra, N. H., 2010. A quasi-decentralized approach for networked state estimation and control of process systems. Ind. Eng. Chem. Res. 49, 7957-7971.

[26] Sun, Y., El-Farra, N. H., 2012. Resource-aware quasi-decentralized control of networked process systems over wireless sensor network. Chem. Eng. Sci. 69, 93-106.

[27] Tetiker, M. D., Artel, F., Cinar, A., 2008. Control of grade transitions in distributed chemical reactor networks: an agent-based approach. Comp. Chem. Eng. 32, 1984-1994.

[28] Tipsuwan, Y., Chow, M.-Y., 2003. Control methodologies in networked control systems. Contr. Eng. Prac. 11, $1099-1111$.

[29] Venkatsubramanian, V., Rengaswamy, R., Yin, K., Kavuri, S. N., 2003. A review of process fault detection and diagnosis - part I: Quantitative model-based methods. Comp. \& Chem. Eng. 27, 293-311.

[30] Yao, Z., El-Farra, N. H., 2013. Resource-aware model predictive control of spatially distributed processes using eventtriggered communication. In: Proceedings of 52nd IEEE Conference on Decision and Control. Florence, Italy, pp. 37263731.

[31] Zhang, Y., Jiang, J., 2008. Bibliographical review on reconfigurable fault-tolerant control systems. Annu. Rev. in Contr. $32,229-252$. 


\section{List of Tables}

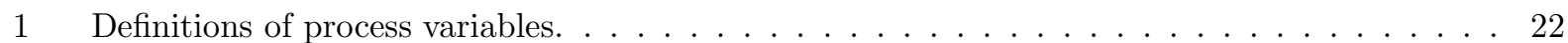

2 Process parameters and unstable steady-state values. . . . . . . . . . . . . . . . 23

3 Estimated fault parameters and their corresponding estimation confidence intervals at differ-

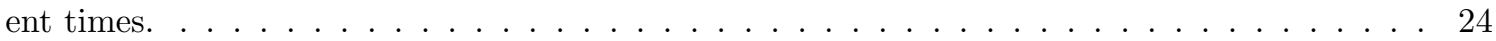




\section{List of Figures}

1 Distributed networked fault identification and accommodation structure for a networked process system. . . . . . . . . . . . . . . . . . . . . . . . . . . 25

2 A schematic showing the time instances at which the moving horizon optimization, measurement transmission, and fault estimation occur in each subsystem. . . . . . . . . . . . . . 26

3 A networked process system involving a cascade of two reactors and a separator with recycle.

4 Plot (a): A contour plot of $\lambda_{\max }(M)$ as a function of the magnitude of the actual fault and the choice of the fault parameter estimate in the control models, with $\alpha_{1}=1$; Plot (b): A contour plot of $\lambda_{\max }(M)$ as a function of the fault size and the controller gain tuning parameter, with $\widehat{\theta}_{1}^{2}=1 \ldots \ldots \ldots \ldots \ldots \ldots \ldots \ldots \ldots \ldots$

5 Plot (a): Evolution of the actual (solid) and estimated (dashed) values of the fault $\theta_{1}$ for the case when a fault with $\theta_{1}=0.9$ occurs at $t=0.33 \mathrm{hr}$. Plot (b): The location of the post-fault operating point with respect to the closed-loop stability region. Plots (c)-(d): Closed-loop state profiles for the first CSTR when an actuator fault with $\theta_{1}=0.9$ occurs at $t=0.33 \mathrm{hr}$ and no fault accommodation is carried out. . . . . . . . . . . . . . . . . .

6 Plot (a): Evolution of the actual (solid) and optimization-based estimated (dashed) values of the fault $\theta_{1}$ for the case when a fault with $\theta_{1}=0.7$ occurs at $t=0.33 \mathrm{hr}$. Plot (b): The locations of the operating point pre and post fault accommodation with respect to the closed-loop stability region. Plots (c)-(d): Closed-loop state profiles for the first CSTR when an actuator fault with $\theta_{1}=0.7$ occurs at $t=0.33 \mathrm{hr}$ and the control model parameters are

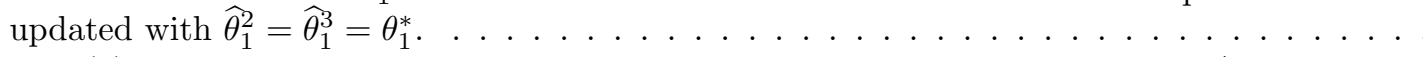

7 Plot (a): The shift in the location of the operating point from point $\mathrm{C}$ to point $\mathrm{C}^{*}$ outside the stability region after the control model parameters are updated. Plot (b): The shift in the location of the operating point from point $\mathrm{C}^{*}$ to point $\mathrm{C}^{* *}$ inside the stability region after the controller gain is adjusted. Plots (c)-(d): The closed-loop state profiles for the first CSTR when an actuator fault with $\theta_{1}=0.3$ occurs at $t=0.33 \mathrm{hr}$ and the control model parameters are updated and the controller gain is adjusted. . . . . . . . . . . . . . . . . .

8 Plot (a): Evolution of the actual (solid) and optimization-based estimated (dashed) values of the fault $\theta_{1}$ for the case when a fault with $\theta_{1}=0.9$ occurs at $t=0.33 \mathrm{hr}$ and $5 \%$ parametric model uncertainty in the heats of reactions is considered. Plot (b): The locations of the fault estimation confidence interval with respect to the closed-loop stability region before $(\mathrm{A})$ and after $\left(\mathrm{A}^{*}\right)$ fault accommodation, with $\alpha_{1}=1$. Plots $(\mathrm{c})-(\mathrm{d})$ : Closed-loop state profiles for the first CSTR when an actuator fault with $\theta_{1}=0.9$ occurs at $t=0.33 \mathrm{hr}$ and the control model parameters are updated with $\widehat{\theta}_{1}^{2}=\widehat{\theta}_{1}^{3}=\theta_{1}^{*}$.

9 Plot (a): The shift in the location of the fault estimation interval from position $\mathrm{D}$ to $\mathrm{D}^{*}$ outside the stability region after the control model parameters are updated, and $\alpha_{1}=1$. Plot (b): The shift in the location of the fault estimation interval from position $\mathrm{D}^{*}$ to position $\mathrm{D}^{* *}$ inside the stability region after the controller gain is adjusted, and $\widehat{\theta}_{1}^{2}=\theta_{1}^{*}$. Plots (c)-(d): The closed-loop state profiles for the first CSTR when an actuator fault with $\theta_{1}=0.5$ occurs at $t=0.33 \mathrm{hr}$ and the control model parameters are updated and the controller gain is adjusted. 33

10 Time evolution profiles of the gradual fault in the actuator of the first CSTR (solid profile), the fault estimate obtained from the online optimization (dashed profile) and the fault estimation interval obtained after each transmission time (dashed vertical lines with circle markers).

11 The sequence of fault accommodation steps taken to compensate for the gradual fault depicted in Fig.10. The uncolored areas in plots (a) and (c) depict the stability regions in the $\left(\theta_{1}, \widehat{\theta}_{1}^{2}\right)$ space for $\alpha_{1}=1$ and $\alpha_{1}=2.5$, respectively. Plots (b) and (d) show the stability regions (uncolored areas) in the $\left(\theta_{1}, \alpha_{1}\right)$ space for $\widehat{\theta}_{1}^{2}=0.4839$ and $\widehat{\theta}_{1}^{2}=0.3548$, respectively. . . . .

12 Evolution of the closed-loop reactant concentration (a) and manipulated input profiles (b) for the first CSTR for case of a gradual fault in the control actuator, subject to the fault accommodation sequence depicted in Fig.11. . . . . . . . . . . . . . . . . . . . . . 36 
13 Time evolution of the actual fault (solid profile) and estimated fault (dashed profile) for the first fault in CSTR 1 (plot (a)) and the second fault in the separator unit (plot (b)). The circle markers represent the bounds on the fault estimation confidence intervals obtained after each transmission time. . . . . . . . . . . . . . . . . . . . . . .

14 Plot (a): A contour plot depicting the closed-loop stability region (uncolored zone) as a function of $\theta_{3}$ and $\widehat{\theta}_{3}$. The red marker indicates the location of the post-fault operating point. Plots (b)-(c): Evolution of the closed-loop temperature, reactant concentration in the first CSTR, and reactant concentration of the separator unit, respectively, for the case when two consecutive faults take place. . . . . . . . . . . . . . . . . . . . . . . 38 
Table 1: Definitions of process variables.

\begin{tabular}{cl}
\hline$C_{A j 0}$ & Concentration of $A$ in the feed stream to tank $j, j=1,2$ \\
$C_{i j}$ & Concentration of species $i, i=A, B, C$ in tank $j, j=1,2,3$ \\
$C_{i r}$ & Concentration of species $i, i=A, B, C$ in the recycle stream \\
$T_{j 0}$ & Temperature of the feed stream to tank $j, j=1,2$ \\
$T_{j}$ & Temperature in tank $j, j=1,2,3$ \\
$T_{r}$ & Temperature in the recycle stream \\
$F_{j 0}$ & Flow rate of the feed stream to tank $j, j=1,2$ \\
$F_{j}$ & Flow rate of the effluent stream from tank $j, j=1,2,3$ \\
$F_{r}$ & Flow rate of the recycle stream \\
$F_{p}$ & Flow rate of the purge stream \\
$V_{j}$ & Volumes of tank $j, j=1,2,3$ \\
$E_{k}$ & Activation energy of reaction $k, k=1,2$ \\
$k_{k}$ & Pre-exponential factor of reaction $k, k=1,2$ \\
$\Delta H_{k}$ & Heat of reaction $k, k=1,2$ \\
$H_{\mathrm{vap}}$ & Heat of vaporization \\
$\alpha_{i}$ & Relative volatilities of species $i, i=A, B, C, D$ \\
$M W_{i}$ & Molecular weights of species $i, i=A, B, C$ \\
$C_{p}$ & Heat capacity \\
$R$ & Gas constant
\end{tabular}


Table 2: Process parameters and unstable steady-state values.

\begin{tabular}{ll}
\hline$T_{10}=300, T_{20}=300$ & $\mathrm{~K}$ \\
$F_{10}=5, F_{20}=5, F_{r}=1.9, F_{p}=0$ & $\mathrm{~m}^{3} / \mathrm{hr}$ \\
$C_{A 10}=4, C_{A 20}=3$ & $\mathrm{kmol} / \mathrm{m}^{3}$ \\
$V_{1}=1.0, V_{2}=0.5, V_{3}=1.0$ & $\mathrm{~m}^{3}$ \\
$E_{1}=5.0 \times 10^{4}, E_{2}=5.5 \times 10^{4}$ & $\mathrm{~kJ} / \mathrm{kmol}$ \\
$k_{1}=3.0 \times 10^{6}, k_{2}=3.0 \times 10^{6}$ & $\mathrm{hr}-1$ \\
$\Delta H_{1}=-5.0 \times 10^{4}, \Delta H_{2}=-5.3 \times 10^{4}$ & $\mathrm{~kJ} / \mathrm{kmol}$ \\
$H_{\text {vap }}=5$ & $\mathrm{~kJ} / \mathrm{kmol}$ \\
$C_{p}=0.231$ & $\mathrm{~kJ} / \mathrm{kg} \cdot \mathrm{K}$ \\
$R=8.314$ & $\mathrm{~kJ} / \mathrm{kmol} \cdot \mathrm{K}$ \\
$\rho=1000$ & $\mathrm{~kg} / \mathrm{m}^{3}$ \\
$\alpha_{A}=2, \alpha_{B}=1, \alpha_{C}=1.5, \alpha_{D}=3$ & $\mathrm{Unitless}$ \\
$M W_{A}=50, M W_{B}=50, M W_{C}=50$ & $\mathrm{~kg} / \mathrm{kmol}$ \\
$T_{1}^{s}=369.5, T_{2}^{s}=435.2, T_{3}^{s}=435.2$ & $\mathrm{~K}$ \\
$C_{A 1}^{s}=3.31, C_{A 2}^{s}=2.75, C_{A 3}^{s}=2.88$ & $\mathrm{kmol} / \mathrm{m}^{3}$ \\
$C_{B 1}^{s}=0.17, C_{B 2}^{s}=0.45, C_{B 3}^{s}=0.50$ & $\mathrm{kmol} / \mathrm{m}^{3}$ \\
$C_{C 1}^{s}=0.04, C_{C 2}^{s}=0.11, C_{C 3}^{s}=0.12$ & $\mathrm{kmol} / \mathrm{m}^{3}$ \\
\hline
\end{tabular}


Table 3: Estimated fault parameters and their corresponding estimation confidence intervals at different times.

\begin{tabular}{cccccc}
\hline Time & $0.205 \mathrm{hr}$ & $0.305 \mathrm{hr}$ & $0.405 \mathrm{hr}$ & $0.505 \mathrm{hr}$ & $0.605 \mathrm{hr}$ \\
\hline$\theta_{1}$ & 0.9516 & 0.752 & 0.4385 & 0.3226 & 0.3031 \\
$\theta_{1}^{*}$ & 0.9795 & 0.8011 & 0.4839 & 0.3548 & 0.317 \\
$\Psi\left(\theta_{1}^{*}\right)$ & $0.9369,0.9768]$ & {$[0.729,0.7856]$} & {$[0.4046,0.4799]$} & {$[0.2946,0.3512]$} & {$[0.2864,0.3202]$} \\
$\widehat{\theta}_{1}^{2}$ & 1 & 0.8011 & 0.4839 & 0.3548 & 0.3548 \\
\hline
\end{tabular}




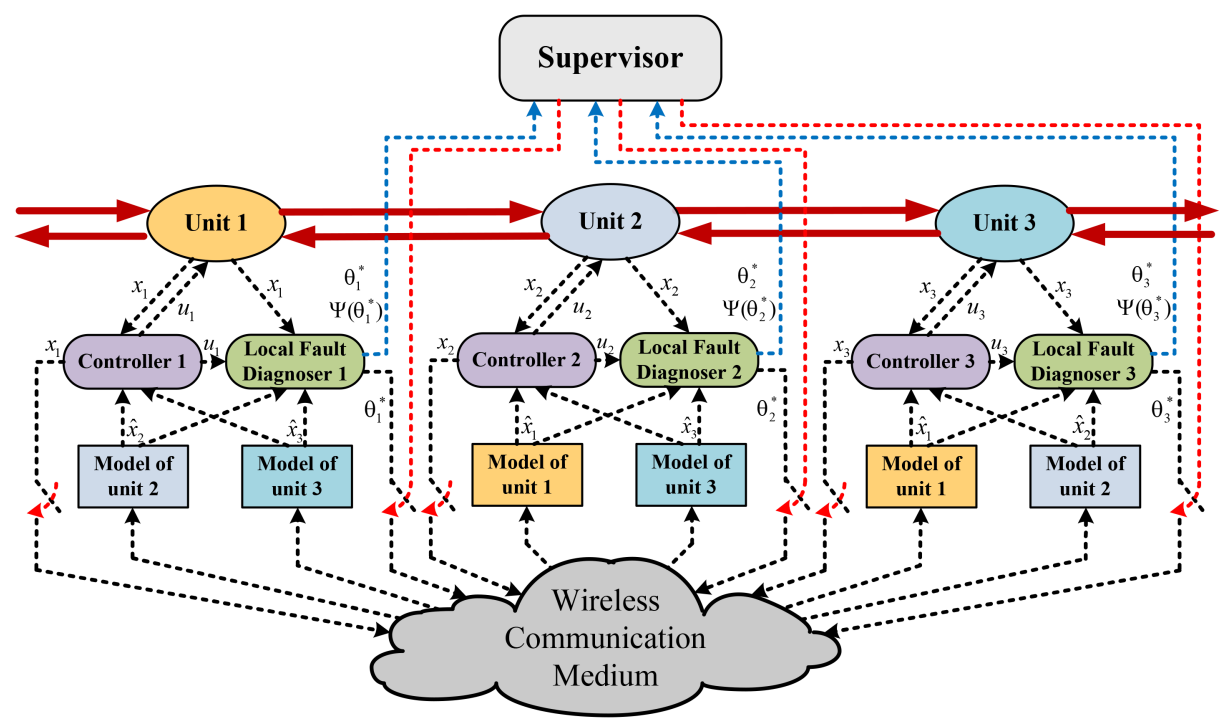

Figure 1: Distributed networked fault identification and accommodation structure for a networked process system. 


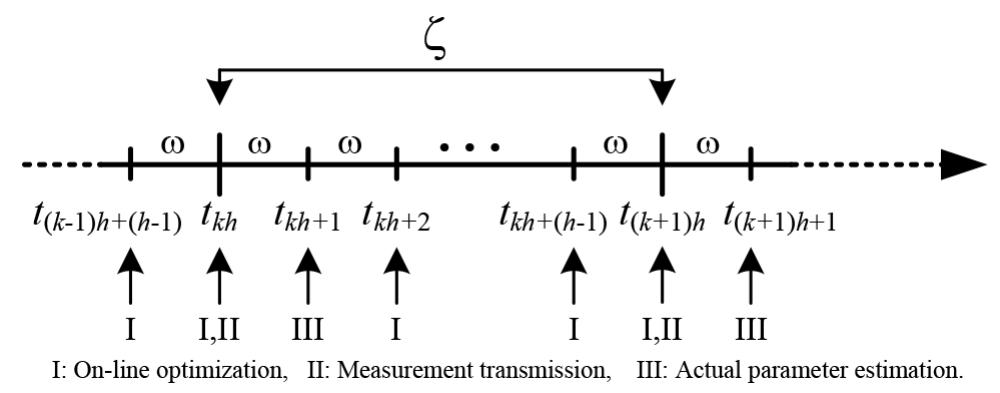

Figure 2: A schematic showing the time instances at which the moving horizon optimization, measurement transmission, and fault estimation occur in each subsystem. 


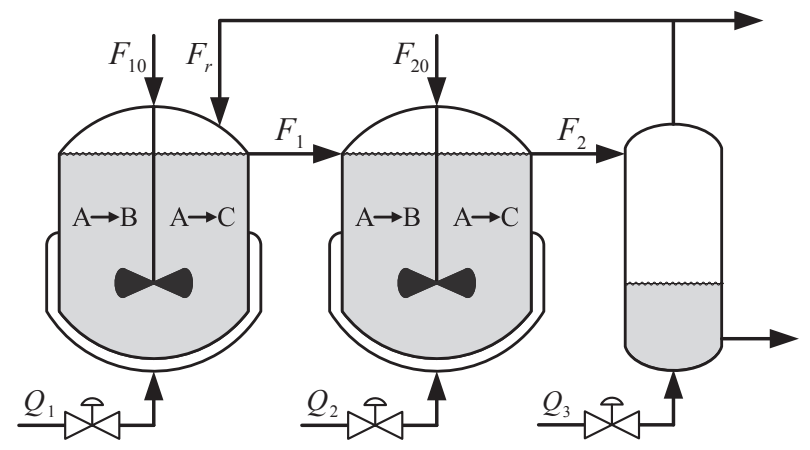

Figure 3: A networked process system involving a cascade of two reactors and a separator with recycle. 

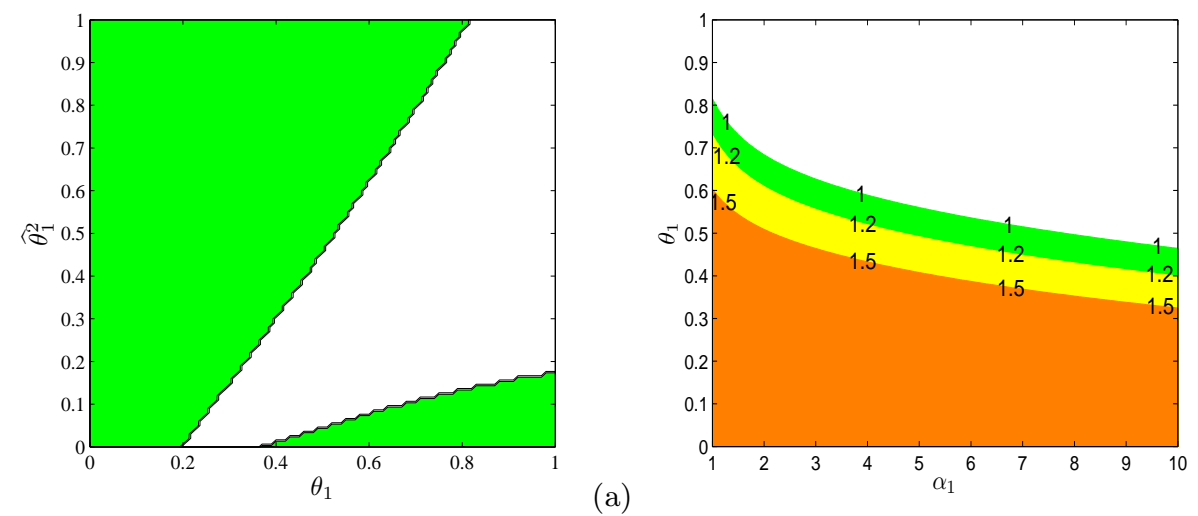

(b)

Figure 4: Plot (a): A contour plot of $\lambda_{\max }(M)$ as a function of the magnitude of the actual fault and the choice of the fault parameter estimate in the control models, with $\alpha_{1}=1$; Plot (b): A contour plot of $\lambda_{\max }(M)$ as a function of the fault size and the controller gain tuning parameter, with $\widehat{\theta}_{1}^{2}=1$ 


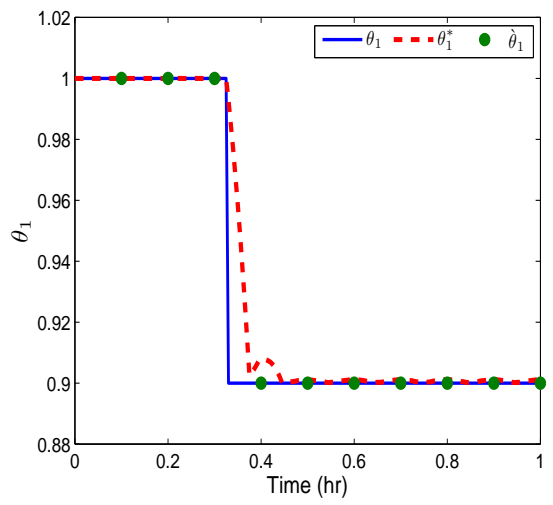

(a)

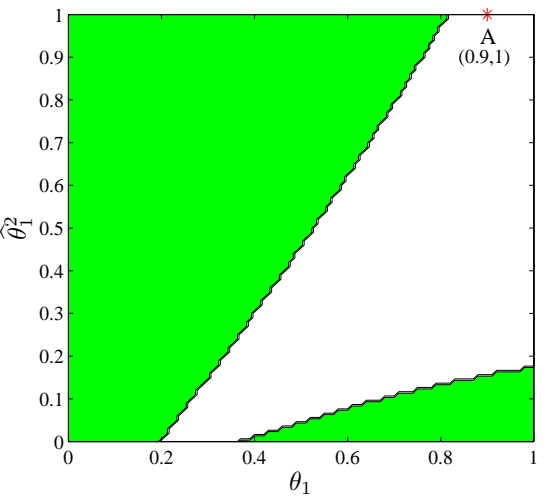

(b)
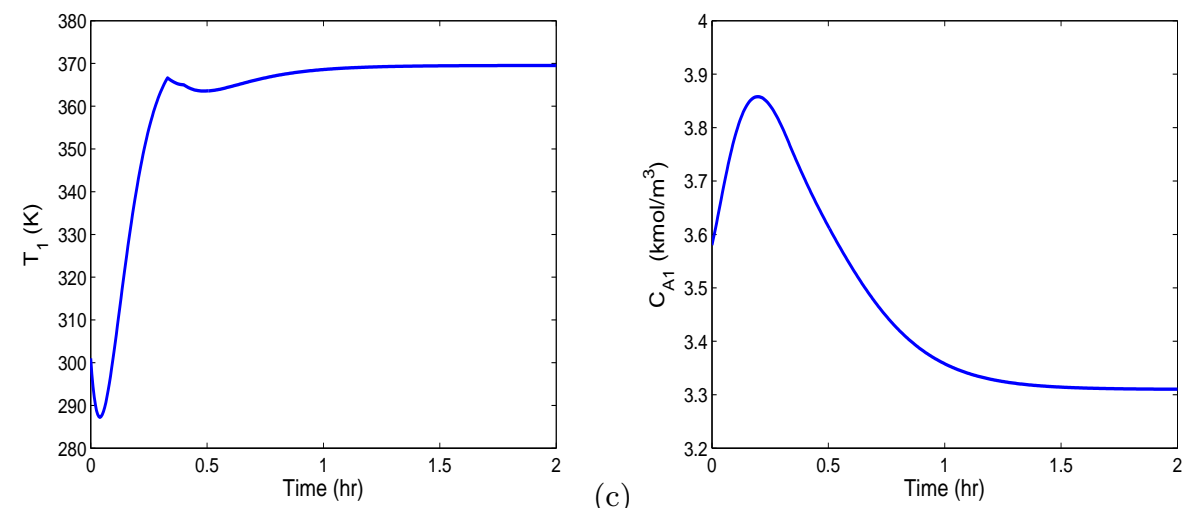

(d)

Figure 5: Plot (a): Evolution of the actual (solid) and estimated (dashed) values of the fault $\theta_{1}$ for the case when a fault with $\theta_{1}=0.9$ occurs at $t=0.33 \mathrm{hr}$. Plot (b): The location of the post-fault operating point with respect to the closed-loop stability region. Plots (c)-(d): Closed-loop state profiles for the first CSTR when an actuator fault with $\theta_{1}=0.9$ occurs at $t=0.33 \mathrm{hr}$ and no fault accommodation is carried out. 

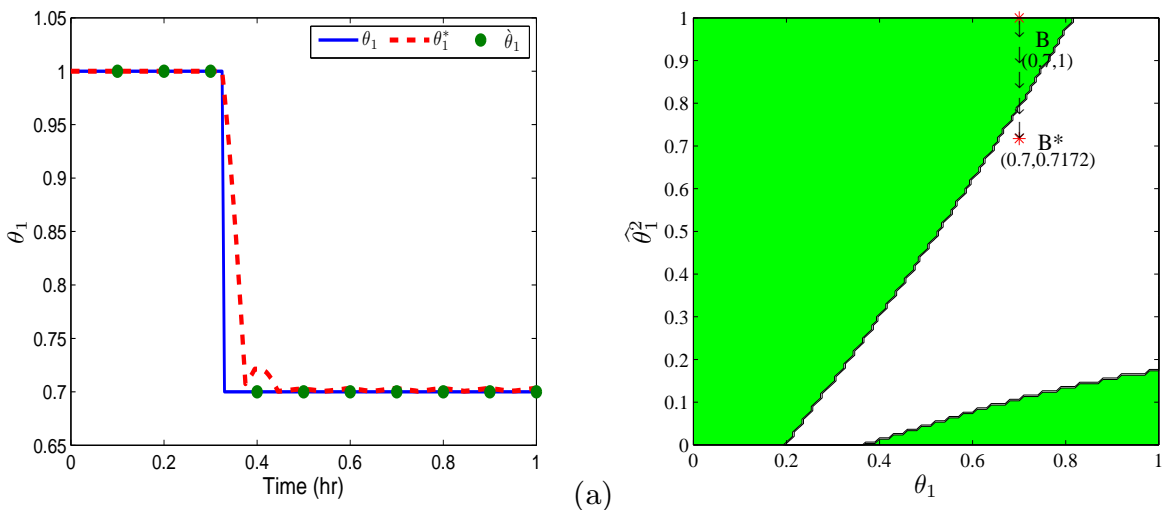

(b)
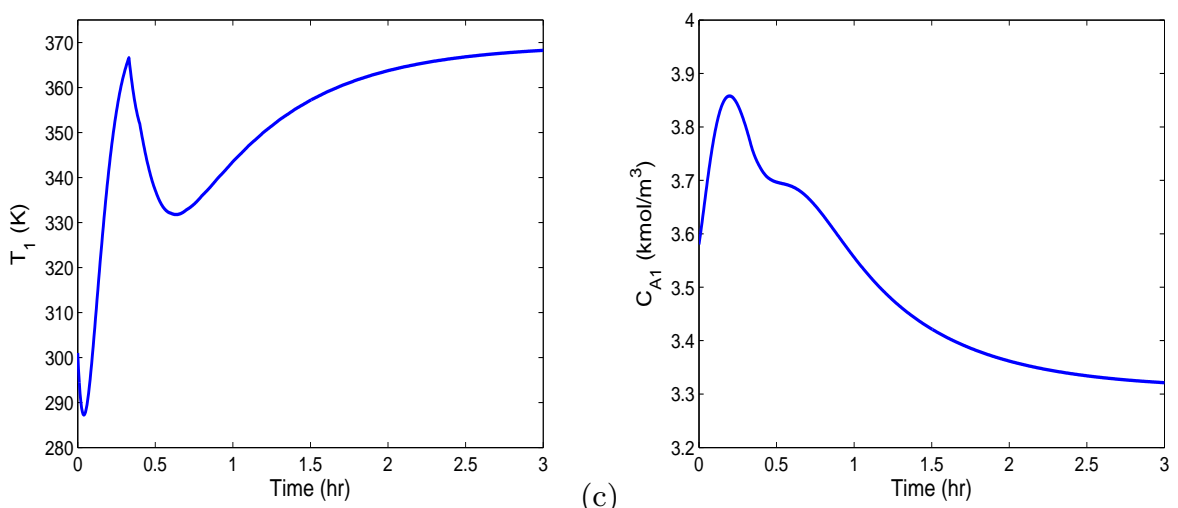

Figure 6: Plot (a): Evolution of the actual (solid) and optimization-based estimated (dashed) values of the fault $\theta_{1}$ for the case when a fault with $\theta_{1}=0.7$ occurs at $t=0.33 \mathrm{hr}$. Plot (b): The locations of the operating point pre and post fault accommodation with respect to the closed-loop stability region. Plots (c)-(d): Closed-loop state profiles for the first CSTR when an actuator fault with $\theta_{1}=0.7$ occurs at $t=0.33 \mathrm{hr}$ and the control model parameters are updated with $\widehat{\theta}_{1}^{2}=\widehat{\theta}_{1}^{3}=\theta_{1}^{*}$. 

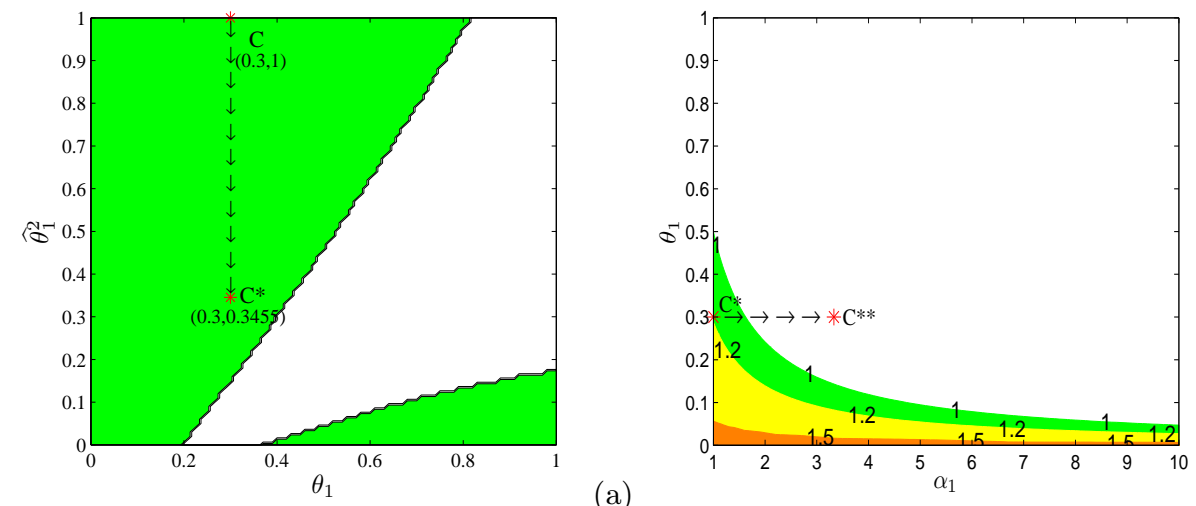

(a)

(b)
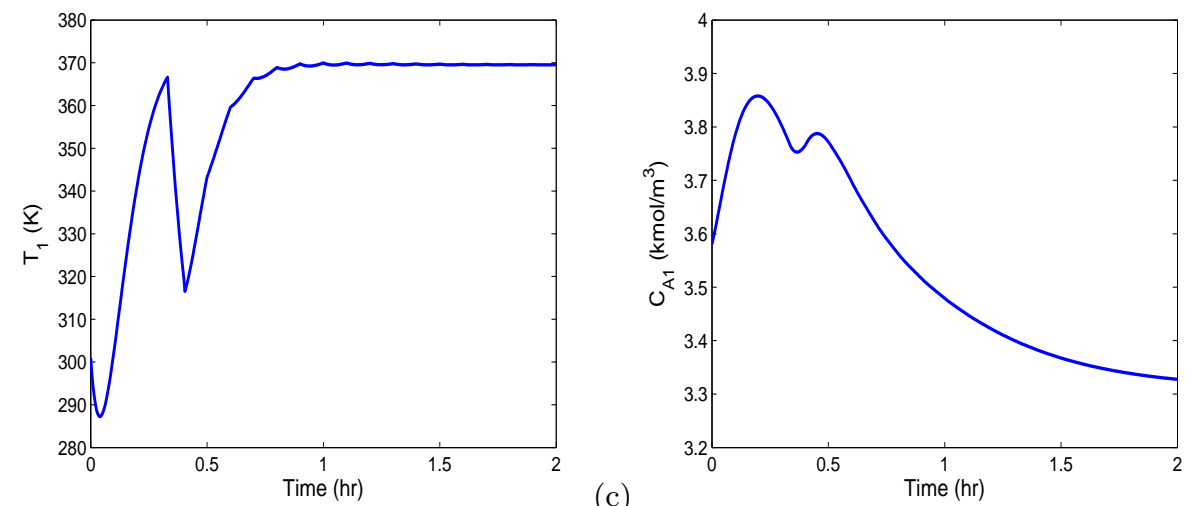

(c)

(d)

Figure 7: Plot (a): The shift in the location of the operating point from point $\mathrm{C}$ to point $\mathrm{C}^{*}$ outside the stability region after the control model parameters are updated. Plot (b): The shift in the location of the operating point from point $\mathrm{C}^{*}$ to point $\mathrm{C}^{* *}$ inside the stability region after the controller gain is adjusted. Plots (c)-(d): The closed-loop state profiles for the first CSTR when an actuator fault with $\theta_{1}=0.3$ occurs at $t=0.33 \mathrm{hr}$ and the control model parameters are updated and the controller gain is adjusted. 

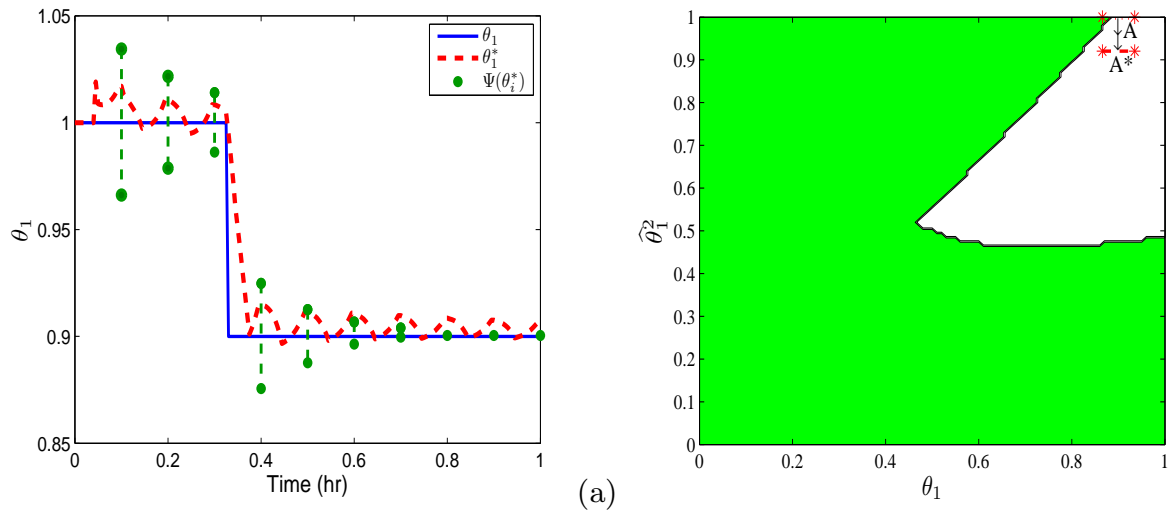

(b)
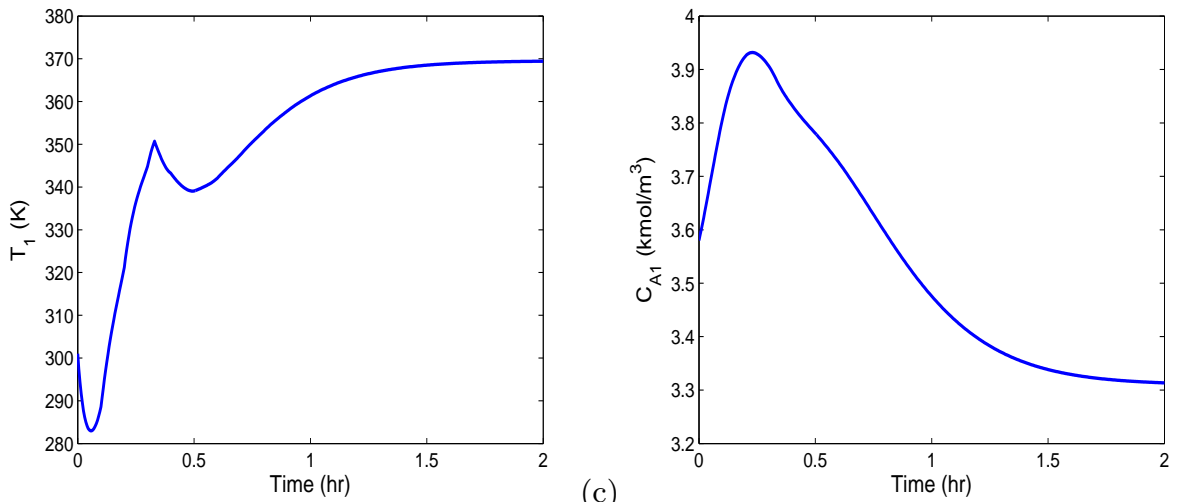

(c)

(d)

Figure 8: Plot (a): Evolution of the actual (solid) and optimization-based estimated (dashed) values of the fault $\theta_{1}$ for the case when a fault with $\theta_{1}=0.9$ occurs at $t=0.33 \mathrm{hr}$ and $5 \%$ parametric model uncertainty in the heats of reactions is considered. Plot (b): The locations of the fault estimation confidence interval with respect to the closed-loop stability region before (A) and after $\left(\mathrm{A}^{*}\right)$ fault accommodation, with $\alpha_{1}=1$. Plots (c)-(d): Closed-loop state profiles for the first CSTR when an actuator fault with $\theta_{1}=0.9$ occurs at $t=0.33 \mathrm{hr}$ and the control model parameters are updated with $\widehat{\theta}_{1}^{2}=\widehat{\theta}_{1}^{3}=\theta_{1}^{*}$. 

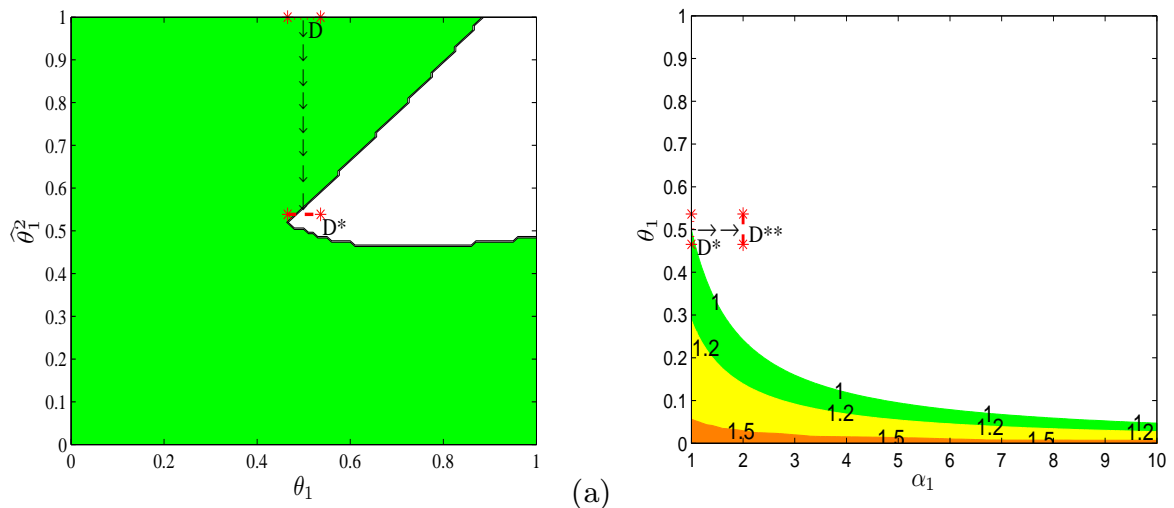

(a)

(b)
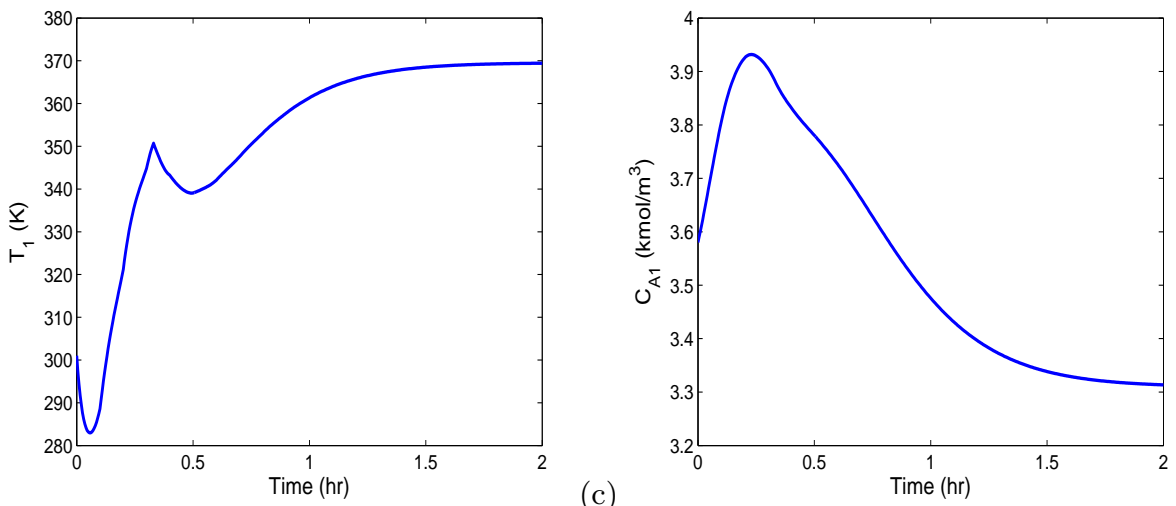

(c)

(d)

Figure 9: Plot (a): The shift in the location of the fault estimation interval from position $\mathrm{D}$ to $\mathrm{D}^{*}$ outside the stability region after the control model parameters are updated, and $\alpha_{1}=1$. Plot (b): The shift in the location of the fault estimation interval from position $\mathrm{D}^{*}$ to position $\mathrm{D}^{* *}$ inside the stability region after the controller gain is adjusted, and $\widehat{\theta}_{1}^{2}=\theta_{1}^{*}$. Plots (c)-(d): The closed-loop state profiles for the first CSTR when an actuator fault with $\theta_{1}=0.5$ occurs at $t=0.33 \mathrm{hr}$ and the control model parameters are updated and the controller gain is adjusted. 


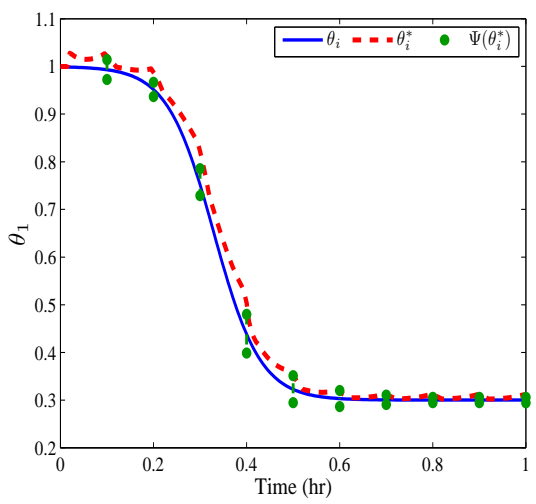

Figure 10: Time evolution profiles of the gradual fault in the actuator of the first CSTR (solid profile), the fault estimate obtained from the online optimization (dashed profile) and the fault estimation interval obtained after each transmission time (dashed vertical lines with circle markers). 

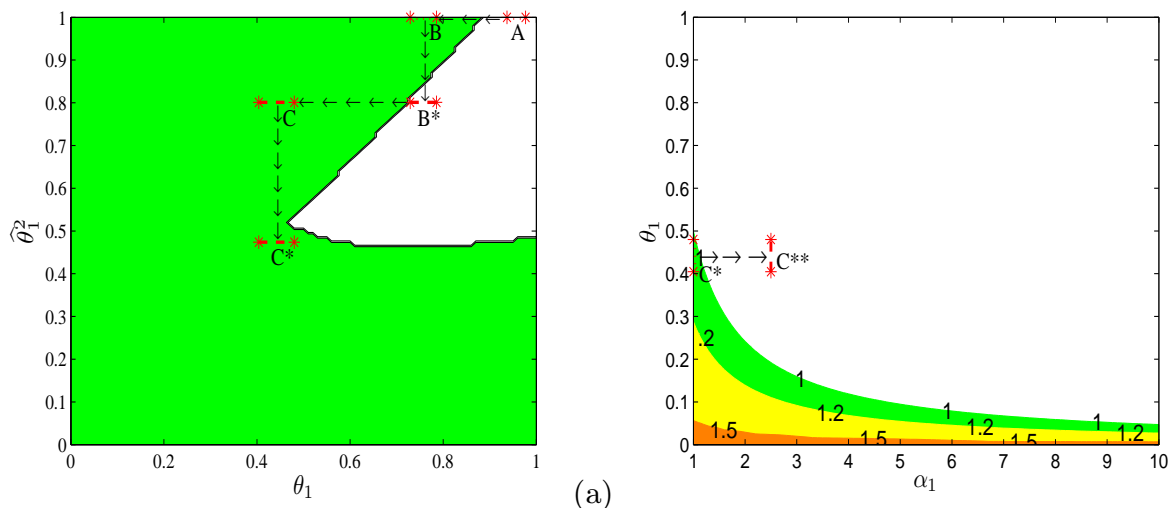

(a)

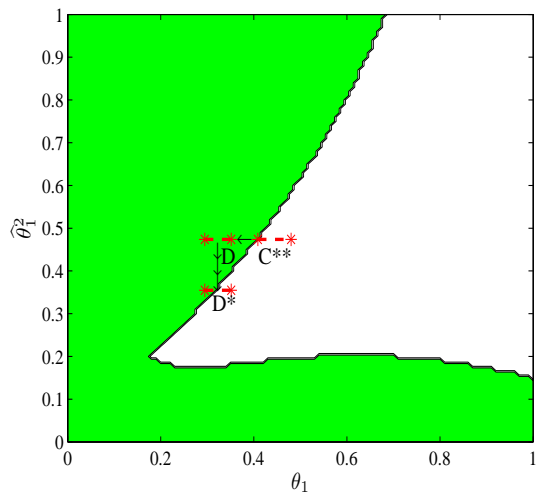

(c)

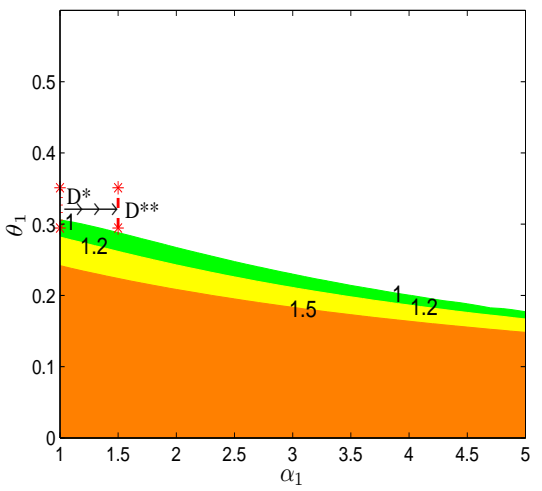

(d)

Figure 11: The sequence of fault accommodation steps taken to compensate for the gradual fault depicted in Fig.10. The uncolored areas in plots (a) and (c) depict the stability regions in the $\left(\theta_{1}, \widehat{\theta}_{1}^{2}\right)$ space for $\alpha_{1}=1$ and $\alpha_{1}=2.5$, respectively. Plots (b) and (d) show the stability regions (uncolored areas) in the $\left(\theta_{1}, \alpha_{1}\right)$ space for $\widehat{\theta}_{1}^{2}=0.4839$ and $\widehat{\theta}_{1}^{2}=0.3548$, respectively. 

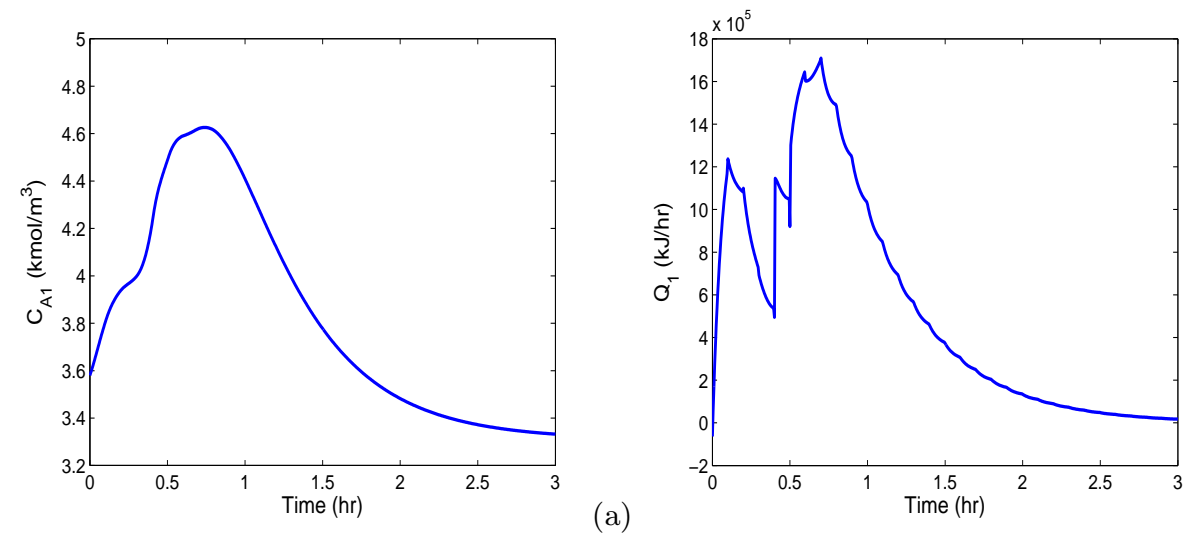

(b)

Figure 12: Evolution of the closed-loop reactant concentration (a) and manipulated input profiles (b) for the first CSTR for case of a gradual fault in the control actuator, subject to the fault accommodation sequence depicted in Fig.11. 

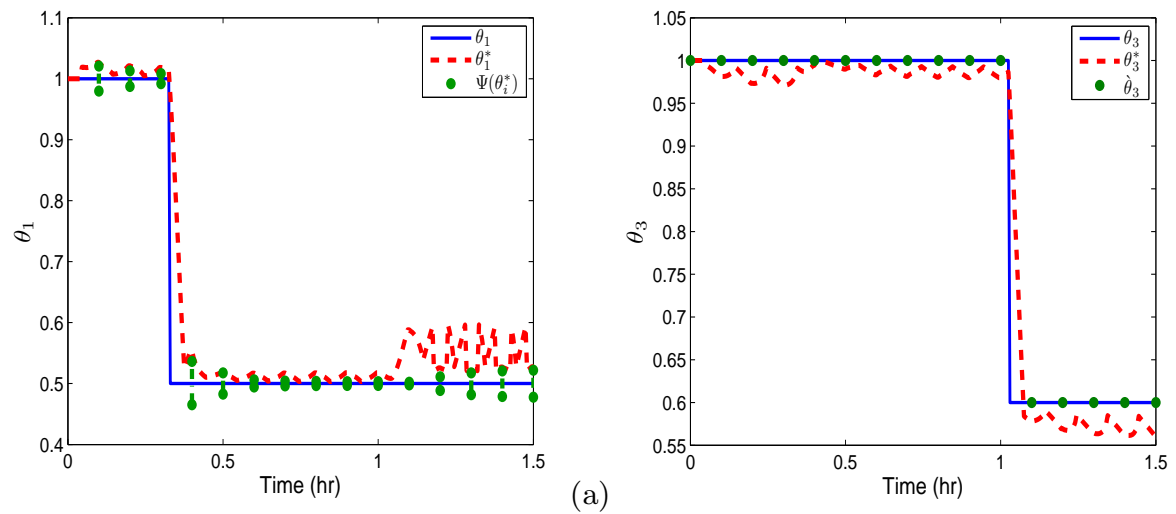

(b)

Figure 13: Time evolution of the actual fault (solid profile) and estimated fault (dashed profile) for the first fault in CSTR 1 (plot (a)) and the second fault in the separator unit (plot (b)). The circle markers represent the bounds on the fault estimation confidence intervals obtained after each transmission time. 

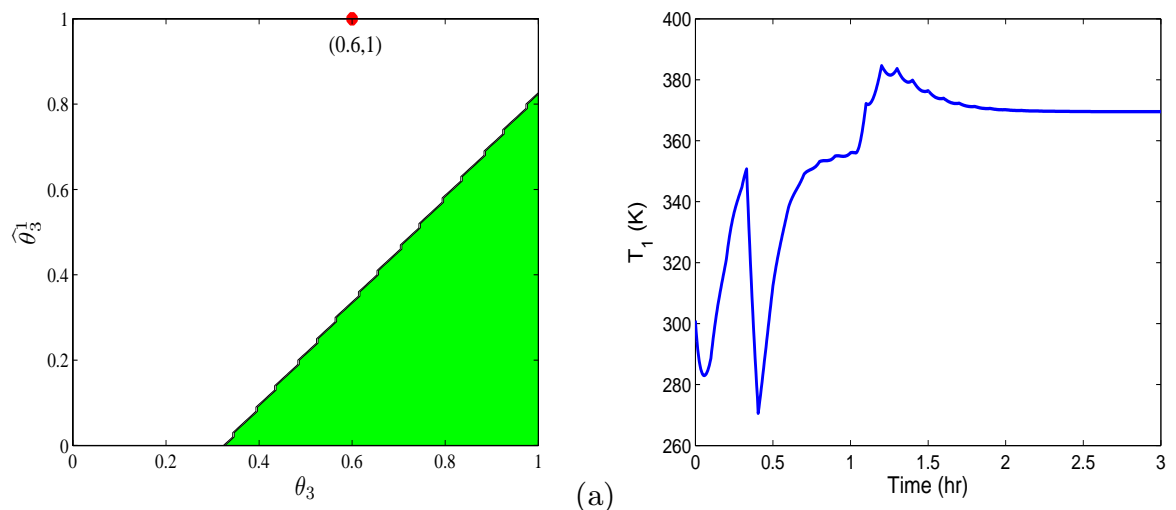

(b)
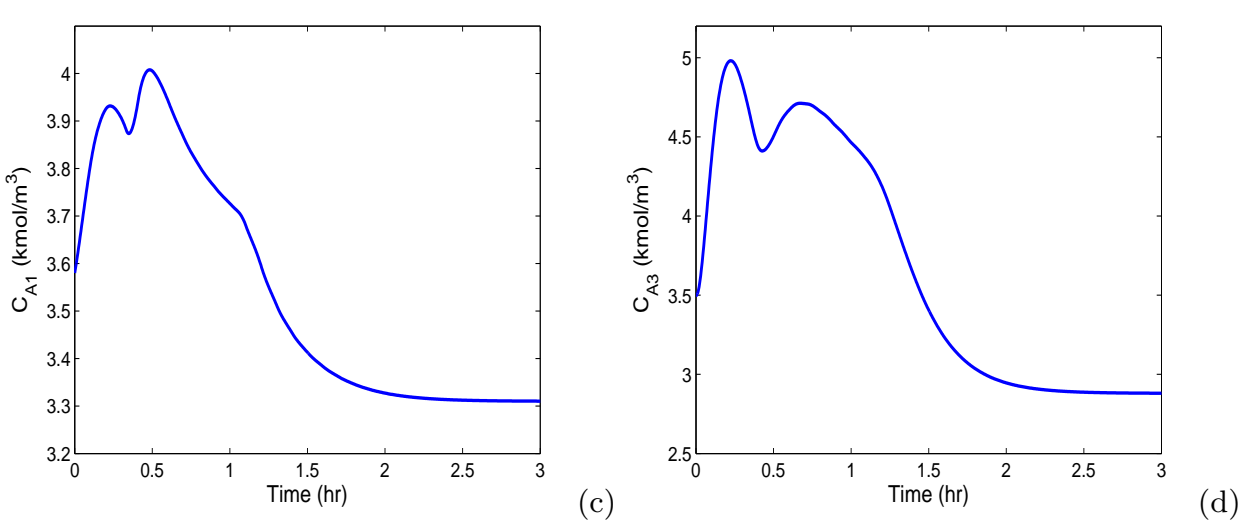

Figure 14: Plot (a): A contour plot depicting the closed-loop stability region (uncolored zone) as a function of $\theta_{3}$ and $\widehat{\theta}_{3}$. The red marker indicates the location of the post-fault operating point. Plots (b)-(c): Evolution of the closed-loop temperature, reactant concentration in the first CSTR, and reactant concentration of the separator unit, respectively, for the case when two consecutive faults take place. 